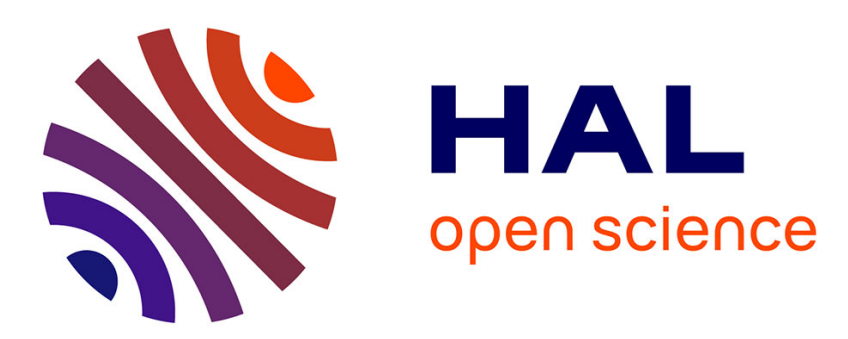

\title{
A General Optimal Multiple Stopping Problem with an Application to Swing Options
}

\author{
Imene Ben Latifa, Joseph Frédéric Bonnans, Mohamed Mnif
}

\section{To cite this version:}

Imene Ben Latifa, Joseph Frédéric Bonnans, Mohamed Mnif. A General Optimal Multiple Stopping Problem with an Application to Swing Options. Stochastic Analysis and Applications, 2015, 33 (4), pp.715-739. 10.1080/07362994.2015.1037592 . hal-01248283

\section{HAL Id: hal-01248283 \\ https://inria.hal.science/hal-01248283}

Submitted on 28 Dec 2015

HAL is a multi-disciplinary open access archive for the deposit and dissemination of scientific research documents, whether they are published or not. The documents may come from teaching and research institutions in France or abroad, or from public or private research centers.
L'archive ouverte pluridisciplinaire HAL, est destinée au dépôt et à la diffusion de documents scientifiques de niveau recherche, publiés ou non, émanant des établissements d'enseignement et de recherche français ou étrangers, des laboratoires publics ou privés. 


\title{
A general optimal multiple stopping problem with an application to Swing Options
}

\author{
Imène Ben Latifa \\ ENIT-LAMSIN, Tunis El Manar University \\ B.P. 37, 1002 Tunis-Belvédère, \\ Tunisie. \\ benlatifa@cmap.polytechnique.fr
}

\author{
Joseph Frédéric Bonnans \\ Ecole Polytechnique-CMAP, \\ 91128 Palaiseau Cedex, \\ France. \\ frederic.bonnans@inria.fr
}

\author{
Mohamed Mnif \\ ENIT-LAMSIN, Tunis El Manar University \\ B.P. 37, 1002 Tunis-Belvédère, \\ Tunisie. \\ mohamed.mnif@enit.rnu.tn
}

April 3, 2015

\begin{abstract}
In their paper [8], Carmona and Touzi have studied an optimal multiple stopping time problem in a market where the price process is continuous. In this paper, we generalize their results when the price process is allowed to jump. Also, we generalize the problem associated to the valuation of swing options to the context of jump diffusion processes. We relate our problem to a sequence of ordinary stopping time problems. We characterize the value function of each ordinary stopping time problem as the unique viscosity solution of the associated Hamilton-Jacobi-Bellman Variational Inequality (HJBVI in short).
\end{abstract}

Key words: Optimal multiple stopping, swing option, jump diffusion process, Snell envelope, viscosity solution. 


\section{Introduction}

Optimal stopping problems in general setting was the object of many works. Maingueneau [19] and El Karoui [13] characterized the optimal stopping time as the beginning of the set where the process is equal to its Snell envelope.

In the Markovian context, Pham [22] studied the valuation of American options when the risky assets are modeled by a jump diffusion process. He showed that the last problem is equivalent to an optimal stopping problem which leads to a parabolic integrodifferential free boundary problem. For details we refer to El Karoui [13], when the reward process is non-negative, right continuous, $\mathbb{F}$-adapted and left continuous in expectation and its supremum is bounded in $L^{p}, p>1$, Karatzas and Shreve [16] in the continuous setting and Peskir and Shiryaev [21] in the Markovian context. Optimal multiple stopping of linear diffusion are investigated in Carmona and Dayanik [7].

Carmona and Touzi [8] introduced the problem of optimal multiple stopping time where the underlying process is continuous. They characterized the optimal multiple stopping time as the solution of a sequence of ordinary stopping time problems. As an application, they studied the valuation of swing options. The latter products are defined as American options with many exercise rights. In fact, the holder of a swing option has the right to exercise it or not at many times under the condition that he respects the refracting time which separates two successive exercises. The consumption in the energy market is not simple, in fact it depends on Foreign parameters like temperature and weather. When the temperature has a high variation, the power consumption has a sharp increase and price follow. Although these spikes of consumption are infrequent, they have a large financial impact, and so the underlying process should be càd-làg. In Dahlgren and Korn [11] the Swing option on the stock market is investigated and a continuous time model for the price of the option in the Black-Scholes framework is derived. Bouzguenda and Mnif [6] generalized the valuation of the swing option where the reward process is allowed to jump. Kobylanski et al. [17] studied an optimal multiple stopping time problem. They showed that such a problem is reduced to compute an optimal one stopping time problem where the new reward function is no longer a right continuous left limited (RCLL) process but a family of positive random variables which satisfy some compatibility properties.

Bender et al.[5] studied the dual representation for generalized multiple stopping problem. They extended the works by Bender (2011a) [1] and Bender (2011b) [2] on multiple exercise options. They take into consideration a refraction period and volume constraints both simultaneously. In the present paper, the holder of the option is restricted to buying at most one package per time and he wait for a refraction period $\delta$. In Bender et al. [5], exercised times are discrete but in our paper they are continuous.

In a general non-Markovian setting, Bender and Dokuchaev [3] studied an optimal control problem related to swing option pricing. They showed that the value process solves a first-order non-linear backward stochastic partial differential equation, and they derived a 
dual minimization problem. In discrete time, Bender and Schoenmakers [4] presented an iterative procedure for solving the multiple stopping problem.

In the present paper, we present a generalization of the classical theory of optimal stopping introduced by El Karoui [13]. We relate our multiple stopping time problem to a sequence of ordinary stopping time problems, we prove the existence of an optimal multiple stopping time. In Bouzguenda and Mnif [6], they assumed that the expectation of the Snell envelope variation is equal to zero for every predictable time, i.e. $E\left[Y_{\tau}^{(i)}-Y_{\tau^{-}}^{(i)}\right]=0$ for any predictable stopping time $\tau$ and for all $i=1, \ldots, \ell$, where $\left(Y_{t}^{(i)}\right)$ is associated to the Snell envelope when the holder of the option exercised $(i-1)$ times. Such assumption is checked when the process is modeled by the exponential of Lévy process. In the present paper, we get rid of this assumption. As in El Karoui [13], we assume that the state process is non-negative, right continuous, $\mathbb{F}$-adapted and left continuous in expectation and its supremum is bounded in $L^{p}, p>1$ and so we can apply our result for a general jump diffusion process. We characterize the value function of each ordinary problem as the unique viscosity solution of the associated HJBVI. Such characterization is important in the sense that if we propose a monotone consistent and stable numerical scheme, then it converges to the unique viscosity solution of the associated HJBVI. This part is postponed in future research. Bouzguenda and Mnif [6] solved numerically the sequence of optimal stopping problem by using Malliavin Calculus to approximate the conditional expectation, but they didn’t obtain a convergence result. In our case such convergence result is possible thanks to the powerful tool of viscosity solutions.

This paper is organized as follows, in section 2 we formulate the problem. In section 3 we provide the existence of a multiple optimal stopping time. In section 4 we study the valuation of swing options in the jump diffusion case. The regularity of the value function is studied in section 5. In the last section we prove that each value function is the unique viscosity solution of the associated HJBVI.

\section{Problem Formulation}

Let $(\Omega, \mathcal{F}, \mathbb{P})$ be a complete probability space, and $\mathbb{F}=\left\{\mathcal{F}_{t}\right\}_{t \geq 0}$ a filtration which satisfies the usual conditions, i.e. an increasing right continuous family of sub $\sigma$-algebras of $\mathbb{F}$ such that $\mathcal{F}_{0}$ contains all the $\mathbb{P}$-null sets. Let $T \in(0, \infty)$ be the option maturity time i.e. the time of expiration of our right to stop the process or exercise, $\mathcal{S}$ the set of $\mathbb{F}$-stopping times with values in $[0, T]$ and $\mathcal{S}_{\sigma}=\{\tau \in \mathcal{S} ; \tau \geq \sigma\}$ for every $\sigma \in \mathcal{S}$.

We shall denote by $\delta>0$ the refraction period which separates two successive exercises. We also fix $\ell \geq 1$ the number of rights we can exercise. Now, we define by $\mathcal{S}_{\sigma}^{(\ell)}$ the set:

$$
\mathcal{S}_{\sigma}^{(\ell)}:=\left\{\begin{array}{l}
\left(\tau_{1}, \ldots, \tau_{\ell}\right) \in \mathcal{S}^{\ell}, \tau_{1} \in \mathcal{S}_{\sigma}, \tau_{i}-\tau_{i-1} \geq \delta \text { on }\left\{\tau_{i-1}+\delta \leq T\right\} \text { a.s, } \\
\tau_{i}=\left(T_{+}\right) \text {on }\left\{\tau_{i-1}+\delta>T\right\} \text { a.s, } \forall i=2, \ldots, \ell,
\end{array}\right\}
$$


where $T_{+}$is a cemetery time. Let $X=\left(X_{t}\right)_{0 \leq t \leq T}$ be a non-negative, right continuous, left continuous in expectation (see definition 2.1 below) and $\mathbb{F}$-adapted process, and assume that $X_{T_{+}}=0$. We recall the definition of left continuous in expectation.

Definition 2.1 A process $X$ is said to be left continuous in expectation (LCE), if for any $\tau \in \mathcal{S}$ and for any sequence $\left(\tau_{n}\right)_{n \geq 0}$ of stopping times such that $\tau_{n} \uparrow \tau$ a.s. one has $\lim _{n \rightarrow \infty} E\left[X_{\tau_{n}}\right]=E\left[X_{\tau}\right]$.

We assume that $X$ satisfies the integrability condition :

$$
E\left[\bar{X}^{p}\right]<\infty \text { for some } p>1 \text {, where } \bar{X}=\sup _{0 \leq t \leq T} X_{t} .
$$

we assume that :

$$
X_{t}=0, \quad \forall t>T \text {. }
$$

We introduce the following optimal multiple stopping problem :

$$
Z_{0}^{(\ell)}:=\sup _{\left(\tau_{1}, \ldots, \tau_{\ell}\right) \in \mathcal{S}_{0}^{(\ell)}} E\left[\sum_{i=1}^{\ell} X_{\tau_{i}}\right] .
$$

It consists in computing the maximum expected reward $Z_{0}^{(\ell)}$ and finding the optimal exercise strategy $\left(\tau_{1}, \ldots, \tau_{\ell}\right) \in \mathcal{S}_{0}^{(\ell)}$ at which the supremum in $(2.4)$ is attained, if such a strategy exists.

Remark 2.1 Notice that Assumption (2.2) guaranties the finiteness of $Z_{0}^{(1)}$. As it is easily seen that $Z_{0}^{(\ell)} \leq \ell Z_{0}^{(1)}$, every $Z_{0}^{(k)}, k \geq 1$, will also be finite.

To solve the optimal multiple stopping problem, we define inductively the sequence :

$$
Y^{(0)}:=0 \quad \text { and } \quad Y_{t}^{(i)}:=\operatorname{ess} \sup _{\tau \in \mathcal{S}_{t}} E\left[X_{\tau}^{(i)} \mid \mathcal{F}_{t}\right], \quad \forall t \geq 0, \quad \forall i=1, \ldots, \ell,
$$

where the i-th exercise reward process $X^{(i)}$ is given by :

$$
X_{t}^{(i)}:=X_{t}+E\left[Y_{t+\delta}^{(i-1)} \mid \mathcal{F}_{t}\right] \quad \text { for } 0 \leq t \leq T-\delta
$$

and

$$
X_{t}^{(i)}:=X_{t} \quad \text { for } t>T-\delta .
$$

Since the paths of $X^{(i)}$ are right continuous, it is known ([13],[19]) that for every stopping time $\theta$

$$
Y_{\theta}^{(i)}=\operatorname{ess} \sup _{\tau \in \mathcal{S}_{\theta}} E\left[X_{\tau}^{(i)} \mid \mathcal{F}_{\theta}\right], \quad \forall i=1, \ldots, \ell
$$

Notation .1 Note that the constants which appear in this paper are generic constants and could change from line to line. 


\section{Existence of an optimal multiple stopping time}

In this section, we shall prove that $Z_{0}^{(\ell)}$ can be computed by solving inductively $\ell$ single optimal stopping problems sequentially. This result is proved in [8] under the assumption that the process $X$ is continuous a.s.. As it is proved by El Karoui [13, Theorem 2.18, p.115], the existence of the optimal stopping strategy for a right continuous, non-negative and $\mathbb{F}$-adapted process $X$ requires assumption (2.2) in addition to the left continuity in expectation (see Definition 2.1) of the process $X$.

Definition 3.1 For all stopping time $\tau$, we said that $\theta^{*} \in \mathcal{S}_{\tau}$ is an optimal stopping time of the problem

$$
Y_{\tau}^{(i)}=e s s \sup _{\theta \in \mathcal{S}_{\tau}} E\left[X_{\theta}^{(i)} \mid \mathcal{F}_{\tau}\right], \quad \forall i=1, \ldots, \ell
$$

if

$$
Y_{\tau}^{(i)}=E\left[X_{\theta^{*}}^{(i)} \mid \mathcal{F}_{\tau}\right] \text { a.s. }
$$

In Lemmas 3.1, 3.2 and Proposition 3.1 we show that the $i$-th exercise reward process $X^{(i)}$ satisfies the conditions required to solve the $i$-th optimal stopping problem.

Lemma 3.1 Suppose that the non-negative $\mathbb{F}$-adapted and right continuous process $X$ satisfies the condition (2.2). Then, for all $i=1, \ldots, \ell$, the process $X^{(i)}$ satisfies :

$$
E\left[\left(\bar{X}^{(i)}\right)^{p}\right]<\infty, \quad p>1, \text { where } \bar{X}^{(i)}:=\sup _{0 \leq t \leq T} X_{t}^{(i)}
$$

Proof. We proceed by induction on $i$.

For $i=1$ we have that $X^{(1)}=X$ so by assumption $(2.2)$ we have that $E\left[\bar{X}^{(1)^{p}}\right]<\infty$.

Let $i \in\{2, \ldots, \ell\}$, let us assume that $E\left[\bar{X}^{(i-1)^{p}}\right]<\infty$, we will show that $E\left[\bar{X}^{(i)^{p}}\right]<\infty$. We have that for all $\tau \in \mathcal{S}_{0}, 0 \leq t \leq T$

$$
E\left[X_{\tau}^{(i-1)} \mid \mathcal{F}_{t}\right] \leq E\left[\sup _{0 \leq s \leq T} X_{s}^{(i-1)} \mid \mathcal{F}_{t}\right]:=\hat{X}_{t}^{(i-1)}
$$

then $Y_{t}^{(i-1)}=\operatorname{ess} \sup _{\tau \in \mathcal{S}_{t}} E\left[X_{\tau}^{(i-1)} \mid \mathcal{F}_{t}\right] \leq \hat{X}_{t}^{(i-1)}$, hence

$$
E\left[\sup _{0 \leq t \leq T}\left(Y_{t}^{(i-1)}\right)^{p}\right] \leq E\left[\sup _{0 \leq t \leq T}\left(\hat{X}_{t}^{(i-1)}\right)^{p}\right]
$$

We have that $\hat{X}^{(i-1)}$ is $\mathbb{F}$-adapted,

$E\left[\hat{X}_{t}^{(i-1)}\right] \leq E\left[E\left[\sup _{0 \leq s \leq T}\left|X_{s}^{(i-1)}\right| \mid \mathcal{F}_{t}\right]\right]=E\left[\sup _{0 \leq s \leq T}\left|X_{s}^{(i-1)}\right|\right]:=E\left[\bar{X}^{(i-1)}\right]<\infty$ and for 
$u \leq t, E\left[\hat{X}_{t}^{(i-1)} \mid \mathcal{F}_{u}\right]=E\left[E\left[\sup _{0 \leq s \leq T} X_{s}^{(i-1)} \mid \mathcal{F}_{t}\right] \mid \mathcal{F}_{u}\right]=E\left[\sup _{0 \leq s \leq T} X_{s}^{(i-1)} \mid \mathcal{F}_{u}\right]:=\hat{X}_{u}^{(i-1)}$, then $\left(\hat{X}_{t}^{(i-1)}\right)$ is a martingale. Hence Doob's $L^{p}$ inequality and Jensen's inequality show:

$$
\begin{aligned}
E\left[\sup _{0 \leq t \leq T}\left(\hat{X}_{t}^{(i-1)}\right)^{p}\right] & \leq\left(\frac{p}{p-1}\right)^{p} \sup _{0 \leq t \leq T} E\left[\left(\hat{X}_{t}^{(i-1)}\right)^{p}\right] \\
& \leq\left(\frac{p}{p-1}\right)^{p} E\left[\left(\bar{X}^{(i-1)}\right)^{p}\right]
\end{aligned}
$$

From (3.2), (3.3) and the induction assumption we deduce that:

$$
E\left[\sup _{0 \leq t \leq T}\left(Y_{t}^{(i-1)}\right)^{p}\right] \leq\left(\frac{p}{p-1}\right)^{p} E\left[\left(\bar{X}^{(i-1)}\right)^{p}\right]<\infty
$$

From inequality (3.4), using the supermartingale property of $Y^{(i-1)}$ and since $\left(\sup _{0 \leq t \leq T} Y_{t}^{(i-1)}\right)^{p}=$ $\sup _{0 \leq t \leq T}\left(Y_{t}^{(i-1)}\right)^{p}$, we obtain

$$
\begin{aligned}
E\left[\left(\bar{X}^{(i)}\right)^{p}\right]^{1 / p} & \leq E\left[\left(\sup _{0 \leq t \leq T}\left(X_{t}+E\left[Y_{t+\delta}^{(i-1)} \mid \mathcal{F}_{t}\right]\right)\right)^{p}\right]^{1 / p} \\
& \leq E\left[\left(\bar{X}+\sup _{0 \leq t \leq T} E\left[Y_{t+\delta}^{(i-1)} \mid \mathcal{F}_{t}\right]\right)^{p}\right]^{1 / p} \\
& \leq C\left(E\left[\bar{X}^{p}\right]^{1 / p}+E\left[\left(\sup _{0 \leq t \leq T} E\left[Y_{t+\delta}^{(i-1)} \mid \mathcal{F}_{t}\right]\right)^{p}\right]^{1 / p}\right) \\
& \leq C\left(E\left[\bar{X}^{p}\right]^{1 / p}+E\left[\sup _{0 \leq t \leq T}\left(Y_{t}^{(i-1)}\right)^{p}\right]^{1 / p}\right)<\infty,
\end{aligned}
$$

where $C$ is a positive constant. Inequality (3.5) implies that: $E\left[\left(\bar{X}^{(i)}\right)^{p}\right]<\infty$.

Lemma 3.2 Suppose that the non-negative $\mathbb{F}$-adapted process $X$ is right continuous, then for all $i=1, \ldots, \ell$, the process $X^{(i)}$ is non-negative $\mathbb{F}$-adapted and right continuous.

Proof. The process $X$ is non-negative and $\mathbb{F}$-adapted, then from the definition of the process $X^{(i)}$ (see equalities (2.6) and (2.7)), we have

$$
\text { for } i=1, \ldots, \ell \text { the process } X^{(i)} \text { is non-negative and } \mathbb{F} \text {-adapted. }
$$

Let us prove by induction that for all $i=1, \ldots, \ell, X^{(i)}$ is right continuous.

For $i=1$ we have that $X^{(1)}=X$, which is right continuous.

Let $i \in\{1, \ldots, \ell-1\}$, assume that $X^{(i)}$ is right continuous and let us prove that $X^{(i+1)}$ is 
right continuous. The process $X$ is right continuous, then from the definition of $X^{(i+1)}$ (equation (3.1)) it suffices to prove that $\left(E\left[Y_{t+\delta}^{(i)} \mid \mathcal{F}_{t}\right]\right)$ is right continuous. To prove this it suffices to prove that $Y^{(i)}$ is right continuous (see Dellacherie and Meyer [12, Theorem 47 p.119]).

Since $X^{(i)}$ is non-negative and $\mathbb{F}$-adapted (from (3.6)), bounded in $L^{1}(\mathbb{P})$ (from Lemma 3.1) and right continuous (from the induction assumption), then from El Karoui [13, Theorem 2.15, p.113] we obtain that $Y^{(i)}$ is right continuous. We deduce then (by Dellacherie and Meyer [12, Theorem 47 p.119]) that the optional projection $\left(E\left[Y_{t+\delta}^{(i)} \mid \mathcal{F}_{t}\right]\right)$ of $Y^{(i)}$ is right continuous and then $X^{(i+1)}$ is also.

We conclude then the right continuity of $X^{(i)}$ for $i=1, \ldots, \ell$.

To prove the existence of optimal stopping time for problem (3.1) we start by giving the definition of a closed under pairwise maximization family and proving that $\left(E\left[X_{\tau}^{(i-1)} \mid \mathcal{F}_{t}\right], \tau \in \mathcal{S}_{t}\right)$ is such a family.

Definition 3.2 A family $\left(X_{i}\right)_{i \in I}$ of random variables is said to be closed under pairwise maximization if for all $i, j \in I$, there exists $k \in I$ such that $X_{k} \geq X_{i} \vee X_{j}$.

Lemma 3.3 Let $t \in[0, T]$, the family $\left(E\left[X_{\tau}^{(i-1)} \mid \mathcal{F}_{t}\right], \tau \in \mathcal{S}_{t}\right)$ is closed under pairwise maximization.

Proof. Let $\tau_{1}, \tau_{2} \in \mathcal{S}_{t}$ and $\tilde{X}_{t, \tau_{j}}^{(i-1)}:=E\left[X_{\tau_{j}}^{(i-1)} \mid \mathcal{F}_{t}\right](j=1,2)$. We define the stopping time

$$
\tau:=\tau_{1} \mathbf{1}_{\left\{\tilde{X}_{t, \tau_{1}}^{(i-1)} \geq \tilde{X}_{t, \tau_{2}}^{(i-1)}\right\}}+\tau_{2} \mathbf{1}_{\left\{\tilde{X}_{t, \tau_{1}}^{(i-1)}<\tilde{X}_{t, \tau_{2}}^{(i-1)}\right\}} .
$$

We have that $\tau \in \mathcal{S}_{t}$ and $E\left[X_{\tau}^{(i-1)} \mid \mathcal{F}_{t}\right] \geq E\left[X_{\tau_{j}}^{(i-1)} \mid \mathcal{F}_{t}\right]$, for $j=1,2$. We deduce then that $\left(E\left[X_{\tau}^{(i-1)} \mid \mathcal{F}_{t}\right], \tau \in \mathcal{S}_{t}\right)$ is closed under pairwise maximization.

This Lemma added to Proposition A.2 p.230 of El Karoui [13] and Theorem 8.1 (see Appendix) gives the following Lemma.

Lemma 3.4 For $i=1, \ldots, \ell$, for all $t \in[0, T]$

$$
E\left[Y_{t}^{(i)}\right]=\sup _{\tau \in \mathcal{S}_{t}} E\left[X_{\tau}^{(i)}\right]
$$

Our aim now is to prove the left continuity in expectation of $X^{(i)}$, for $i=1, \ldots, \ell$. In the following proposition we prove that $X^{(i)}$ is LCE.

Proposition 3.1 Suppose that the non-negative $\mathbb{F}$-adapted process $X$ is right continuous, left continuous in expectation ( $L C E$ ) and satisfies condition $(2.2)$. Then, for all $i=1, \ldots, \ell$, the process $X^{(i)}$ is LCE. 
Proof. We proceed by induction.

For $i=1$, we have that $X^{(1)}=X$, so it is left continuous in expectation.

Let $1 \leq i \leq \ell-1$, assume that $X^{(i)}$ is LCE and we will show that $X^{(i+1)}$ is LCE.

We begin by proving that $Y^{(i)}$ is LCE.

Let $\tau \in \mathcal{S}$ and $\left(\tau_{n}\right)$ be a sequence of stopping times such that $\tau_{n} \uparrow \tau$ a.s.. Note that by the supermartingale property of $Y^{(i)}$ we have

$$
E\left[Y_{\tau_{n}}^{(i)}\right] \geq E\left[Y_{\tau}^{(i)}\right], \quad \forall n \in \mathbb{N} .
$$

Since $X^{(i)}$ is non-negative, $\mathbb{F}$-adapted, bounded in $L^{1}(\mathbb{P})$ and LCE, then by El Karoui [13]

$$
\theta_{n}^{(i)}=\inf \left\{t \geq \tau_{n}, X_{t}^{(i)}=Y_{t}^{(i)}\right\}
$$

is an optimal stopping time of

$$
Y_{\tau_{n}}^{(i)}=\operatorname{ess} \sup _{\tau \in \mathcal{S}_{\tau_{n}}} E\left[X_{\tau}^{(i)} \mid \mathcal{F}_{\tau_{n}}\right]
$$

So by the Definition 3.1, we obtain that

$$
E\left[Y_{\tau_{n}}^{(i)}\right]=E\left[X_{\theta_{n}^{(i)}}^{(i)}\right]
$$

Moreover, it is clear that $\left(\theta_{n}^{(i)}\right)_{i}$ is a nondecreasing sequence of stopping times dominated by $T$. Let us define $\bar{\theta}^{(i)}:=\lim _{n \rightarrow \infty} \uparrow \theta_{n}^{(i)}$. Note that $\bar{\theta}^{(i)}$ is a stopping time. Also, as for each $n, \theta_{n}^{(i)} \geq \tau_{n}$ a.s., it follows that $\bar{\theta}^{(i)} \in \mathcal{S}_{\tau}$. Therefore, since $X^{(i)}$ is LCE and since $\theta_{n}^{(i)}$ is an optimal stopping time of (3.9), we obtain

$$
\begin{aligned}
E\left[Y_{\tau}^{(i)}\right] & =\sup _{\theta \in \mathcal{S}_{\tau}} E\left[X_{\theta}^{(i)}\right] \\
& \geq E\left[X_{\bar{\theta}^{(i)}}^{(i)}\right] \\
& =\lim _{n \rightarrow \infty} E\left[X_{\theta_{n}^{(i)}}^{(i)}\right] \\
& =\lim _{n \rightarrow \infty} E\left[Y_{\tau_{n}}^{(i)}\right] .
\end{aligned}
$$

By inequalities (3.8) and (3.10) we deduce that $Y^{(i)}$ is LCE.

Let $\tau$ be a stopping time and $\left(\tau_{n}\right)_{n}$ a sequence of stopping times such that $\tau_{n} \uparrow \tau$ a.s., we have that

$$
E\left[X_{\tau_{n}}^{(i+1)}\right]=E\left[X_{\tau_{n}}\right]+E\left[Y_{\tau_{n}+\delta}^{(i)}\right]
$$

Sending $n$ to $\infty$, we obtain that $E\left[X_{\tau_{n}}^{(i+1)}\right] \rightarrow E\left[X_{\tau}^{(i+1)}\right]$, and then $X^{(i+1)}$ is LCE. We conclude then that for all $i=1, \ldots, \ell, X^{(i)}$ is LCE.

Let us set:

$$
\tau_{1}^{*}:=\inf \left\{t \geq 0 ; Y_{t}^{(\ell)}=X_{t}^{(\ell)}\right\}
$$


Since $Y_{T}^{(\ell)}=X_{T}^{(\ell)}$, then $\tau_{1}^{*} \leq T$ a.s.. Next, for $2 \leq i \leq \ell$, we define

$$
\tau_{i}^{*}:=\inf \left\{t \geq \delta+\tau_{i-1}^{*} ; Y_{t}^{(\ell-i+1)}=X_{t}^{(\ell-i+1)}\right\} \mathbf{1}_{\left\{\delta+\tau_{i-1}^{*} \leq T\right\}}+\left(T_{+}\right) \mathbf{1}_{\left\{\delta+\tau_{i-1}^{*}>T\right\}}
$$

Clearly, $\vec{\tau}^{*}:=\left(\tau_{1}^{*}, \ldots, \tau_{\ell}^{*}\right) \in \mathcal{S}_{0}^{(\ell)}$.

Since for all $i=1, \ldots, \ell, X^{(\ell-i+1)}$ is a non-negative right continuous $\mathbb{F}$-adapted process that satisfies the integrability condition $E\left[\right.$ ess $\left.\sup _{\tau \in \mathcal{S}} X_{\tau}^{(\ell-i+1)}\right]<\infty$ and which is LCE along stopping times, then we obtain the existence of optimal stopping time (see El Karoui [13, Theorem 2.18, p.115], Theorem 8.2 and Proposition 8.2 in the Appendix). We can then cite the following Theorem.

Theorem 3.1 (Existence of optimal stopping time) For each $\tau \in \mathcal{S}$ there exists an optimal stopping time of the problem

$$
Y_{\tau}^{(\ell-i+1)}=e s s \sup _{\theta \in \mathcal{S}_{\tau}} E\left[X_{\theta}^{(\ell-i+1)} \mid \mathcal{F}_{\tau}\right], \quad \forall i=1, \ldots, \ell .
$$

Moreover $\tau_{i}^{*}$ is the minimal optimal stopping time of the problem

$$
Y_{\tau_{i-1}^{*}+\delta}^{(\ell-i+1)}=e s s \sup _{\tau \in \mathcal{S}_{\tau_{i-1}^{*}+\delta}^{*}} E\left[X_{\tau}^{(\ell-i+1)} \mid \mathcal{F}_{\tau_{i-1}^{*}+\delta}\right], \quad \forall i=1, \ldots, \ell .
$$

( by convention $\tau_{0}^{*}+\delta=0$ ).

We have also that

$$
E\left[Y_{\tau_{i}^{*}}^{(\ell-i+1)}\right]=\sup _{\tau \in \mathcal{S}_{\tau_{i-1}^{*}+\delta}^{*}} E\left[X_{\tau}^{(\ell-i+1)}\right]
$$

and the stopped supermartingale $\left\{Y_{t \wedge \tau_{i}^{*}}^{(\ell-i+1)}, \tau_{i-1}^{*}+\delta \leq t \leq T\right\}$ is a martingale.

By Theorem 3.2 we generalize Theorem 1 of [8] to right-continuous price processes.

Theorem 3.2 Let us assume that the non-negative, $\mathbb{F}$-adapted process $X$ is right continuous, left continuous in expectation and satisfies condition (2.2). Then,

$$
Z_{0}^{(\ell)}=Y_{0}^{(\ell)}=E\left[\sum_{i=1}^{\ell} X_{\tau_{i}^{*}}\right],
$$

where $\left(\tau_{1}^{*}, \ldots, \tau_{\ell}^{*}\right)$ represents the optimal exercise strategy.

Proof. From Theorem 3.1, $\tau_{i}^{*}$ is an optimal stopping time for the problem

$$
Y_{\tau_{i-1}^{*}+\delta}^{(\ell-i+1)}=\operatorname{ess} \sup _{\tau \in \mathcal{S}_{\tau_{i-1}^{*}+\delta}^{*}} E\left[X_{\tau}^{(\ell-i+1)} \mid \mathcal{F}_{\tau_{i-1}^{*}+\delta}\right]
$$

Let $\vec{\tau}=\left(\tau_{1}, \ldots, \tau_{\ell}\right)$ be an arbitrary element in $\mathcal{S}_{0}^{(\ell)}$. For ease of notation, we set $\bar{\tau}_{i}:=\tau_{\ell-i+1}$. 
A) Let us prove that, for all $1 \leq i \leq \ell$

$$
E\left[\sum_{j=1}^{\ell} X_{\tau_{j}}\right] \leq E\left[X_{\bar{\tau}_{i}}^{(i)}+\sum_{j=i+1}^{\ell} X_{\bar{\tau}_{j}}\right]
$$

We prove this result by induction. For $i=1$ we have that

$$
E\left[\sum_{j=1}^{\ell} X_{\tau_{j}}\right] \leq E\left[X_{\bar{\tau}_{1}}^{(1)}+\sum_{j=2}^{\ell} X_{\bar{\tau}_{j}}\right]
$$

since $X^{(1)} \equiv X$ and for all $\ell \geq 1$ we have $\sum_{j=1}^{\ell} X_{\tau_{j}}=\sum_{j=1}^{\ell} X_{\bar{\tau}_{j}}$. We conclude then that the inequality (3.14) is true for $i=1$.

Let $1 \leq i \leq \ell-1$, suppose that (3.14) is true for $i$. We prove that it is true for $i+1$. We have that

$$
\begin{aligned}
E\left[\sum_{j=1}^{\ell} X_{\tau_{j}}\right] & =E\left[\sum_{j=1}^{\ell} X_{\bar{\tau}_{j}}\right] \\
& =E\left[\sum_{j=1}^{i} X_{\bar{\tau}_{j}}+\sum_{j=i+1}^{\ell} X_{\bar{\tau}_{j}}\right],
\end{aligned}
$$

so by the assumption (3.14) we have that

$$
E\left[X_{\bar{\tau}_{i}}^{(i)}\right] \geq E\left[\sum_{j=1}^{i} X_{\bar{\tau}_{j}}\right]
$$

Let us prove that

$$
E\left[X_{\bar{\tau}_{i}}^{(i)}\right] \leq E\left[X_{\bar{\tau}_{i+1}}^{(i+1)}-X_{\bar{\tau}_{i+1}}\right]
$$

- If $T-\delta<\bar{\tau}_{i+1} \leq T$,

then $X_{\bar{\tau}_{i+1}}^{(i+1)}=X_{\bar{\tau}_{i+1}}$. We have that $\tau_{\ell-i}=\bar{\tau}_{i+1}>T-\delta$ so $\bar{\tau}_{i}=\tau_{\ell-i+1} \geq \delta+\tau_{\ell-i}>T$, then $X_{\bar{\tau}_{i}}^{(i)}=0$, see assumption (2.3), and then

$$
E\left[X_{\bar{\tau}_{i}}^{(i)}\right]=0=E\left[X_{\bar{\tau}_{i+1}}^{(i+1)}-X_{\bar{\tau}_{i+1}}\right] .
$$

- If $0 \leq \bar{\tau}_{i+1} \leq T-\delta$,

we have that $\bar{\tau}_{i}=\tau_{\ell-i+1} \geq \tau_{\ell-i}+\delta=\bar{\tau}_{i+1}+\delta$, then $\bar{\tau}_{i} \in \mathcal{S}_{\bar{\tau}_{i+1}+\delta}$, which implies

$$
\begin{aligned}
E\left[X_{\bar{\tau}_{i}}^{(i)}\right] & =E\left[E\left[X_{\bar{\tau}_{i}}^{(i)} \mid \mathcal{F}_{\bar{\tau}_{i+1}+\delta}\right]\right] \\
& \leq E\left[Y_{\bar{\tau}_{i+1}+\delta}^{(i)}\right] \\
& =E\left[X_{\bar{\tau}_{i+1}}^{(i+1)}-X_{\bar{\tau}_{i+1}}\right] .
\end{aligned}
$$


Then the inequality (3.17) holds in both cases.

In view of (3.17) and in addition with (3.16) it gives that

$$
E\left[\sum_{j=1}^{i} X_{\bar{\tau}_{j}}\right] \leq E\left[X_{\bar{\tau}_{i+1}}^{(i+1)}-X_{\bar{\tau}_{i+1}}\right] .
$$

In addition with (3.15) we obtain that

$$
\begin{aligned}
E\left[\sum_{j=1}^{\ell} X_{\tau_{j}}\right] & \leq E\left[X_{\bar{\tau}_{i+1}}^{(i+1)}\right]+E\left[\sum_{j=i+1}^{\ell} X_{\bar{\tau}_{j}}\right]-E\left[X_{\bar{\tau}_{i+1}}\right] \\
& =E\left[X_{\bar{\tau}_{i+1}}^{(i+1)}\right]+E\left[\sum_{j=i+2}^{\ell} X_{\bar{\tau}_{j}}\right]
\end{aligned}
$$

and then (3.14) is true for $i+1$. We conclude then that (3.14) is true for all $0 \leq i \leq \ell$.

B) Now using (3.14) with $i=\ell$, the definition of $Y_{0}^{(\ell)}$ and Theorem 3.1, we can see that:

$$
E\left[\sum_{j=1}^{\ell} X_{\tau_{j}}\right] \leq E\left[X_{\tau_{1}}^{(\ell)}\right] \leq Y_{0}^{(\ell)}=E\left[X_{\tau_{1}^{*}}^{(\ell)}\right] .
$$

We have that $E\left[X_{\tau_{1}^{*}}^{(\ell)}\right]=E\left[X_{\tau_{1}^{*}}+E\left[Y_{\tau_{1}^{*}+\delta}^{(\ell-1)} \mid \mathcal{F}_{\tau_{1}^{*}}\right]\right]$, then

$$
E\left[\sum_{j=1}^{\ell} X_{\tau_{j}}\right] \leq Y_{0}^{(\ell)}=E\left[X_{\tau_{1}^{*}}\right]+E\left[Y_{\tau_{1}^{*}+\delta}^{(\ell-1)}\right] .
$$

By Theorem 3.1 and equation (2.6) we have that for all $i=1, \ldots, \ell-1$

$$
\begin{aligned}
E\left[Y_{\tau_{i}^{*}+\delta}^{(\ell-i)}\right] & =E\left[X_{\tau_{i+1}^{*}}^{(\ell-i)}\right] \\
& =E\left[X_{\tau_{i+1}^{*}}\right]+E\left[Y_{\tau_{i+1}^{*}+\delta}^{(\ell-i-1)}\right]
\end{aligned}
$$

By equations (3.21), (3.22) and the assumption that $Y^{(0)}=0$, we obtain that

$$
E\left[\sum_{i=1}^{\ell} X_{\tau_{i}}\right] \leq Y_{0}^{(\ell)} \leq E\left[X_{\tau_{1}^{*}}+\ldots+X_{\tau_{\ell}^{*}}\right]
$$

We have that $\left(\tau_{1}, \ldots, \tau_{\ell}\right)$ is an arbitrary element in $\mathcal{S}_{0}^{(\ell)}$ so

$$
Z_{0}^{(\ell)}=\sup _{\tau \in \mathcal{S}_{0}^{(\ell)}} E\left[\sum_{i=1}^{\ell} X_{\tau_{i}}\right] \leq Y_{0}^{(\ell)} \leq E\left[X_{\tau_{1}^{*}}+\ldots+X_{\tau_{\ell}^{*}}\right] .
$$

By the definition of $Z_{0}^{(\ell)}$ we have that $E\left[X_{\tau_{1}^{*}}+\ldots+X_{\tau_{\ell}^{*}}\right] \leq Z_{0}^{(\ell)}$, which joined to inequality (3.23) prove the optimality of the stopping times vector $\left(\tau_{1}^{*}, \ldots, \tau_{\ell}^{*}\right)$ for the problem $Z_{0}^{(\ell)}$ together with the equality $Z_{0}^{(\ell)}=Y_{0}^{(\ell)}$. 


\section{Swing Options in the jump diffusion Model}

In this section, we consider a jump diffusion model. We prove that conditions ensuring the existence of an optimal stopping time vector for the optimal multiple stopping time problem are satisfied. Then, we give the solution to the valuation and a vector of optimal stopping times of the swing option under the risk neutral probability measure for general jump diffusion processes.

\subsection{The jump diffusion Model}

We consider two assets $\left(S^{0}, X\right)$, where $S^{0}$ is the bond and $X$ is a risky asset. The dynamics of $S^{0}$ is given by $d S_{t}^{0}=r S_{t}^{0} d t$, where $r>0$ is the interest rate. We assume that the financial market is incomplete, so there are many equivalent martingale measures. We denote by $Q$ an equivalent martingale measure, and the expectation under $Q$ by $E^{Q}$.

We define under $Q$, an $\mathbb{F}$-standard Brownian motion $W$ and an $\mathbb{F}$-homogeneous Poisson random measure $v$ with intensity measure $q(d s, d z)=d s \times m(d z), m$ is the Lévy measure on $\mathbb{R}$ of $v$ and $\tilde{v}(d s, d z):=(v-q)(d s, d z)$ is called the compensated jump martingale random measure of $v$. The process $X=\left(X_{t}\right)_{0 \leq t \leq T}$ evolves according to the following stochastic differential equation:

$$
d X_{s}=b\left(s, X_{s^{-}}\right) d s+\sigma\left(s, X_{s^{-}}\right) d W_{s}+\int_{\mathbb{R} \backslash\{0\}} \gamma\left(s, X_{s^{-}}, z\right) \tilde{v}(d s, d z), \quad X_{t}=x,
$$

where $b, \sigma$, and $\gamma$ are continuous functions with respect to $(t, x)$. The Lévy measure $m$ is a positive, $\sigma$-finite measure on $\mathbb{R}$, such that

$$
\int_{|z| \geq 1} m(d z)<+\infty .
$$

Furthermore, we shall make the following assumptions:

there exists $K>0, \rho: \mathbb{R} \rightarrow \mathbb{R}_{+}$, with

$$
\int_{\mathbb{R} \backslash\{0\}} \rho^{2}(z) m(d z)<\infty,
$$

such that for all $t \in[0, T], x, y$ and $z \in \mathbb{R}$,

$$
\begin{aligned}
|b(t, x)-b(t, y)|+|\sigma(t, x)-\sigma(t, y)| & \leq K|x-y| \\
|\gamma(t, x, z)-\gamma(t, y, z)| & \leq \rho(z)|x-y| \\
|\gamma(t, x, z)| & \leq \rho(z)(1+|x|) .
\end{aligned}
$$

Notice that the continuity of $b, \sigma$ and $\gamma$ with respect to $(t, x)$ and the Lipschitz condition (4.4) implies the global linear condition

$$
|b(t, x)|+|\sigma(t, x)| \leq K(1+|x|)
$$


Assumptions on $b, \sigma$ and $\gamma$ ensure that there exists a unique càdlàg adapted solution to (4.1) such that

$$
E^{Q}\left[\sup _{s \in[0, T]}\left|X_{s}\right|^{2}\right]<\infty
$$

(see Øksendal and Sulem [20]). We shall also use the notation $X_{s}^{t, x}$ for $X_{s}$ whenever we need to emphasize the dependence of the process $X$ on its initial condition.

Let us give an example for which these technical conditions, especially on the jump component, are satisfied.

Example $4.1 m$ is a finite measure on $\mathbb{R}: m(d z)=\lambda h(z) d z$, and $h$ is a probability density which admits second order moment. $\gamma(t, x, z)=x z$ so that assumptions (4.5)(4.6) are satisfied with $\rho(z)=z$.

Remark 4.1 The process $X$ solution of (4.1) is quasi-left continuous (see [25, Chapter III]). It means that $\Delta X_{\tau}=0$ a.s on the set $\{\tau<\infty\}$, for every predictable time $\tau$.

\subsection{Formulation of the Optimal Multiple Stopping Time Problem}

The value function of the swing option problem with $\ell$ exercise rights and refraction time $\delta>0$ is given by:

$$
v^{(\ell)}(x):=v^{(\ell)}(0, x):=\sup _{\left(\tau_{1}, \ldots, \tau_{\ell}\right) \in \mathcal{S}_{0}^{(\ell)}} E^{Q}\left[\sum_{i=1}^{\ell} e^{-r \tau_{i}} \phi\left(X_{\tau_{i}}^{0, x}\right)\right],
$$

where $\phi: \mathbb{R} \longrightarrow \mathbb{R}^{+}$is a Lipschitz payoff function with linear growth.

To solve the problem (4.8), we need to define the dynamic version:

$$
v^{(k)}(t, x):=\operatorname{ess} \sup _{\tau \in \mathcal{S}_{t}} E^{Q}\left[e^{-r(\tau-t)} \phi^{(k)}\left(\tau, X_{\tau}\right) \mid X_{t}=x\right], \quad v^{(0)} \equiv 0
$$

where $k=1, \ldots, \ell$ and

$$
\begin{aligned}
& \phi^{(k)}(t, x):=\phi(x)+e^{-r \delta} E^{Q}\left[v^{(k-1)}\left(t+\delta, X_{t+\delta}\right) \mid X_{t}=x\right], \quad \forall 0 \leq t \leq T-\delta \\
& \phi^{(k)}(t, x):=\phi(x), \quad \forall T-\delta<t \leq T .
\end{aligned}
$$

Assume that for all $s>T, \phi^{(k)}\left(s, X_{s}\right)=0$.

It is well-known that the process $\left(e^{-r s} v^{(k)}\left(s, X_{s}\right)\right)_{s \in[0, T]}$ is the Snell envelope of the process $\left(e^{-r s} \phi^{(k)}\left(s, X_{s}\right)\right)_{s \in[0, T]}$. To apply the general result, Theorem 3.2, on the optimal multiple stopping time problem obtained in the previous section, we have to show that section 2 conditions are satisfied. This is proved in Proposition 4.1, where the reward process is then given by $U_{s}:=e^{-r s} \phi\left(X_{s}\right)$. 
Proposition 4.1 The $\mathbb{F}$-adapted process $U$ satisfies the following conditions :

$$
\begin{aligned}
& U \text { is right continuous, } \\
& E^{Q}\left[\bar{U}^{2}\right]<\infty \text { where } \bar{U}=\sup _{0 \leq s \leq T} U_{s}, \\
& U \text { is left continuous in expectation, }
\end{aligned}
$$

Proof. Since $\phi$ is a continuous function and $X$ is right continuous, then the process $\left(U_{s}\right)_{s \in[0, T]}=\left(e^{-r s} \phi\left(X_{s}\right)\right)_{s \in[0, T]}$ is right continuous.

From the growth condition of $\phi$, we have

$$
\forall 0 \leq s \leq T, \quad U_{s} \leq K\left(1+\left|X_{s}\right|\right) \text { a.s. } \quad \text { and } \quad E^{Q}\left[\left|X_{s}\right|^{2}\right] \leq E^{Q}\left[\sup _{0 \leq u \leq T}\left|X_{u}\right|^{2}\right]<\infty,(
$$

then $E^{Q}\left[\bar{U}^{2}\right]<\infty$.

Since $X$ is quasi left continuous, then from the continuity of the payoff function $\phi$ we deduce that it is also the case for the process $U$. By the dominated convergence theorem, we deduce that $\lim _{n \rightarrow \infty} E^{Q}\left[U_{\tau_{n}}\right]=E^{Q}\left[U_{\tau}\right]$ and then $U$ is left continuous in expectation.

Let us set

$$
\theta_{1}^{*}:=\inf \left\{s \geq 0 ; v^{(\ell)}\left(s, X_{s}\right)=\phi^{(\ell)}\left(s, X_{s}\right)\right\}
$$

For $2 \leq k \leq \ell$, we define

$\theta_{k}^{*}:=\inf \left\{s \geq \delta+\theta_{k-1}^{*} ; v^{(\ell-k+1)}\left(s, X_{s}\right)=\phi^{(\ell-k+1)}\left(s, X_{s}\right)\right\} \mathbf{1}_{\left\{\delta+\theta_{k-1}^{*} \leq T\right\}}+\left(T_{+}\right) \mathbf{1}_{\left\{\delta+\theta_{k-1}^{*}>T\right\}}$.

Our aim is to relate problem (4.8) to the sequence of problems defined in (4.9). The next theorem allows us to use Theorem 3.1 in the Markovian context.

Theorem 4.1 For each $k=1, \ldots, \ell$, there exists an optimal stopping time for problem (4.9). Moreover $\theta_{k}^{*}$ is the minimal optimal stopping time of the problem

$$
v^{(\ell-k+1)}\left(\theta_{k-1}^{*}+\delta, X_{\theta_{k-1}^{*}+\delta}\right)=e s s \sup _{\tau \in \mathcal{S}_{\theta_{k-1}^{*}+\delta}} E^{Q}\left[e^{-r\left(\tau-\theta_{k-1}^{*}-\delta\right)} \phi^{(\ell-k+1)}\left(\tau, X_{\tau}\right) \mid \mathcal{F}_{\theta_{k-1}^{*}}+\delta\right] .
$$

Also we have

$$
v^{(\ell)}(x)=E^{Q}\left[\sum_{i=1}^{\ell} e^{-r \theta_{i}^{*}} \phi\left(X_{\theta_{i}^{*}}\right)\right] .
$$

Proof. By Proposition 4.1 we have that $U$ is left continuous in expectation and satisfies $E^{Q}\left[\bar{U}^{2}\right]<\infty$, so by Proposition 3.1 we obtain that for each $k=1, \ldots, \ell, U^{(k)}:=$ $\left(e^{-r s} \phi^{(k)}\left(s, X_{s}\right)\right)$ is also left continuous in expectation. In addition, it is a non negative right continuous $\mathbb{F}$-adapted process and satisfies $E^{Q}\left[\left(\bar{U}^{(k)}\right)^{2}\right]<\infty$, see Lemma 3.1. By Theorem 3.1 we obtain the first required result, and by Theorem 3.2, we obtain the second. 


\section{Properties of the value functions}

In this section we study the regularity of the sequence of the payoff functions defined by (4.10) and the sequence of value functions defined by (4.9).

Lemma 5.1 For all $k=1, \ldots, \ell$, there exists $K>0$ such that for all $(t, x) \in[0, T] \times \mathbb{R}$

$$
\left|\phi^{(k)}(t, x)\right| \leq K(1+|x|) \quad \text { and } \quad\left|v^{(k)}(t, x)\right| \leq K(1+|x|) .
$$

Proof. We proceed by induction on $k$.

For $k=1$, by the linear growth of $\phi$ we have that

$$
\phi^{(1)}(t, x):=\phi(t, x) \leq K(1+|x|)
$$

and by Lemma 3.1 of Pham [23, p.9]

$$
v^{(1)}(t, x) \leq K(1+|x|) .
$$

Let $1 \leq k \leq \ell-1$, suppose that there exists $K>0$ such that for all $(t, x) \in[0, T] \times \mathbb{R}$, $\left|\phi^{(k)}(t, x)\right| \leq K(1+|x|)$ and $\left|v^{(k)}(t, x)\right| \leq K(1+|x|)$, then

$$
\begin{aligned}
\phi^{(k+1)}(t, x) & =\phi(x)+e^{-r \delta} E^{Q}\left[v^{(k)}\left(t+\delta, X_{t+\delta}^{t, x}\right)\right] \\
& \leq K(1+|x|) .
\end{aligned}
$$

Then by Lemma 3.1 of Pham [23, p.9] we obtain that

$$
\begin{aligned}
v^{(k+1)}(t, x) & =\sup _{\tau \in \mathcal{S}_{t}} E^{Q}\left[e^{-r(\tau-t)} \phi^{(k)}\left(X_{\tau}^{t, x}\right)\right] \\
& \leq K(1+|x|) .
\end{aligned}
$$

Which proves the desired result.

Proposition 5.1 For all $k=1, \ldots, \ell$, there exists $K>0$ such that for all $t \in[0, T]$, $x, y \in \mathbb{R}$

$$
\left|\phi^{(k)}(t, x)-\phi^{(k)}(t, y)\right| \leq K|x-y| \quad \text { and } \quad\left|v^{(k)}(t, x)-v^{(k)}(t, y)\right| \leq K|x-y| .
$$

Proof. We proceed by induction on $k$.

For $k=1$, we have that $\phi^{(1)}:=\phi$ which is Lipschitz and

$$
\begin{aligned}
\left|v^{(1)}(t, x)-v^{(1)}(t, y)\right| & \leq \sup _{\tau \in \mathcal{S}_{t}} E^{Q}\left[\left|\phi\left(X_{\tau}^{t, x}\right)-\phi\left(X_{\tau}^{t, y}\right)\right|\right] \\
& \leq K|x-y| .
\end{aligned}
$$


Let $1 \leq k \leq \ell-1$, suppose that there exists $K>0$ such that for all $t \in[0, T], x, y \in \mathbb{R}$, $\left|\phi^{(k)}(t, x)-\phi^{(k)}(t, y)\right| \leq K|x-y|$ and $\left|v^{(k)}(t, x)-v^{(k)}(t, y)\right| \leq K|x-y|$, then

$$
\begin{aligned}
\left|\phi^{(k+1)}(t, x)-\phi^{(k+1)}(t, y)\right| & \leq|\phi(x)-\phi(y)|+K e^{-r \delta} E^{Q}\left[\left|X_{t+\delta}^{t, x}-X_{t+\delta}^{t, y}\right|\right] \\
& \leq K|x-y|,
\end{aligned}
$$

so $\phi^{(k+1)}$ is Lipschitz with respect to $x$. Let us prove that it is also for $v^{(k+1)}$, we have

$$
\begin{aligned}
\left|v^{(k+1)}(t, x)-v^{(k+1)}(t, y)\right| & \leq \sup _{\tau \in \mathcal{S}_{t}} E^{Q}\left[\left|\phi^{(k+1)}\left(\tau, X_{\tau}^{t, x}\right)-\phi^{(k+1)}\left(\tau, X_{\tau}^{t, y}\right)\right|\right] \\
& \leq K|x-y|
\end{aligned}
$$

then $v^{(k+1)}$ is Lipschitz with respect to $x$.

We conclude then that for all $k=1, \ldots, \ell, \phi^{(k)}$ and $v^{(k)}$ are both Lipschitz with respect to $x$.

To prove the following theorem, we need to recall the Dynamic Programming Principle.

Proposition 5.2 [23](Dynamic Programming Principle) For all $(t, x) \in[0, T] \times \mathbb{R}$, $h \in \mathcal{S}_{t}, k=1, \ldots, \ell$ we have

$$
v^{(k)}(t, x)=\sup _{\tau \in \mathcal{S}_{t}} E^{Q}\left[\mathbf{1}_{\{\tau<h\}} e^{-r(\tau-t)} \phi^{(k)}\left(\tau, X_{\tau}^{t, x}\right)+\mathbf{1}_{\{\tau \geq h\}} e^{-r(h-t)} v^{(k)}\left(h, X_{h}^{t, x}\right)\right] .
$$

Theorem 5.1 There exists a constant $C>0$ such that, for $k=1, \ldots, \ell$, for all $t<s \in$ $[0, T], x \in \mathbb{R}$,

$$
\left|\phi^{(k)}(t, x)-\phi^{(k)}(s, x)\right| \leq C(1+|x|) \sqrt{s-t}
$$

and

$$
\left|v^{(k)}(s, x)-v^{(k)}(t, x)\right| \leq C(1+|x|) \sqrt{s-t} .
$$

Proof. Let us prove this theorem by induction.

For $k=1$, we have that $\phi^{(1)}(t, x)=\phi^{(1)}(s, x)=\phi(x)$, then the inequality (5.7) is true for $k=1$.

Let $0 \leq t<s \leq T$, by the Dynamic Programming Principle, the Lipschitz property of $\phi$ and $v^{(1)}$, Lemma 3.1 of Pham [23] and from the growth condition of $v^{(1)}$ we obtain that

$$
\left|v^{(1)}(t, x)-v^{(1)}(s, x)\right| \leq C(1+|x|) \sqrt{s-t} .
$$

Let $1 \leq k \leq \ell-1$, suppose that (5.7) and (5.8) are true for $k$ and let us prove that there are true for $k+1$.

By the induction assumption and Lemma 8.1 (see Appendix) we obtain

$$
\left|\phi^{(k+1)}(t, x)-\phi^{(k+1)}(s, x)\right| \leq C(1+|x|) \sqrt{s-t} .
$$


By the Dynamic Programming Principle, the Lipschitz property of $\phi^{(k+1)}$ and $v^{(k+1)}$, Lemma 3.1 of Pham [23] and from the growth condition of $v^{(k+1)}$ we obtain that

$$
\left|v^{(k+1)}(t, x)-v^{(k+1)}(s, x)\right| \leq C(1+|x|) \sqrt{s-t} .
$$

We can then conclude that (5.7) and (5.8) are true for all $k=1, \ldots, \ell$.

\section{Viscosity solutions and comparison theorem}

The aim of this section is to characterize the value function as the unique viscosity solution of the associated HJBVI defined by

$$
\begin{gathered}
\min \left\{r v^{(k)}(t, x)-\frac{\partial v^{(k)}}{\partial t}(t, x)-A\left(t, x, \frac{\partial v^{(k)}}{\partial x}(t, x), \frac{\partial^{2} v^{(k)}}{\partial x^{2}}(t, x)\right)-B\left(t, x, \frac{\partial v^{(k)}}{\partial x}(t, x), v^{(k)}\right) ;\right. \\
\left.v^{(k)}(t, x)-\phi^{(k)}(t, x)\right\}=0, \quad \forall(t, x) \in[0, T) \times \mathbb{R}
\end{gathered}
$$

where, $\phi^{(k)}$ is introduced in (4.10) and for $t \in[0, T], x \in \mathbb{R}, p \in \mathbb{R}, M \in \mathbb{R}$ the operator:

$$
A(t, x, p, M):=\frac{1}{2} \sigma^{2}(t, x) M+b(t, x) p .
$$

For $\varphi \in \mathcal{C}^{1,2}([0, T] \times \mathbb{R}) \cap \mathcal{C}_{2}([0, T] \times \mathbb{R})$, we define:

$$
B\left(t, x, \frac{\partial \varphi}{\partial x}(t, x), \varphi\right):=\int_{\mathbb{R} \backslash\{0\}}\left[\varphi(t, x+\gamma(t, x, z))-\varphi(t, x)-\gamma(t, x, z) \frac{\partial \varphi}{\partial x}(t, x)\right] m(d z),
$$

where

$$
\mathcal{C}_{2}([0, T] \times \mathbb{R}):=\left\{\varphi \in \mathcal{C}^{0}([0, T] \times \mathbb{R}) / \sup _{[0, T] \times \mathbb{R}} \frac{|\varphi(t, x)|}{1+|x|^{2}}<+\infty\right\} .
$$

Let us prove that $B$ is well defined. For $t \in[0, T], x \in \mathbb{R}, p \in \mathbb{R}$ and $\varphi \in \mathcal{C}^{1,2}([0, T] \times \mathbb{R})$, we can define:

$$
B_{1}(t, x, \varphi):=\int_{0<|z| \leq 1}\left[\varphi(t, x+\gamma(t, x, z))-\varphi(t, x)-\gamma(t, x, z) \frac{\partial \varphi}{\partial x}(t, x)\right] m(d z) .
$$

This integral term can be written also:

$$
B_{1}(t, x, \varphi)=\int_{0<|z| \leq 1} \gamma^{2}(t, x, z) \int_{0}^{1}\left(1-y \frac{\partial^{2} \varphi}{\partial^{2} x}(t, x+y \gamma(t, x, z)) d y m(d z) .\right.
$$

From inequality (4.6) and the regularity of $\varphi$ we obtain that the integrand of $B_{1}(t, x, \varphi)$ is bounded by $C_{t, x} \rho^{2}(z)$. Then from assumption (4.3) we prove that $B_{1}$ is well defined. We define also for $\varphi \in \mathcal{C}_{2}([0, T] \times \mathbb{R})$ :

$$
B^{1}(t, x, p, \varphi):=\int_{|z| \geq 1}[\varphi(t, x+\gamma(t, x, z))-\varphi(t, x)-\gamma(t, x, z) p] m(d z) .
$$


The integrand of $B^{1}(t, x, p, \varphi)$ is bounded by $C_{p, x}\left(1+|\gamma(t, x, z)|^{2}\right)$ and then from assumptions (4.2) on $m$ and (4.6) on $\gamma$ this integral term is convergent.

We conclude that the integrodifferential operator $B$ is well defined for all $\varphi \in \mathcal{C}^{1,2}([0, T] \times$ $\mathbb{R}) \cap \mathcal{C}_{2}([0, T] \times \mathbb{R})$ and $B\left(t, x, \frac{\partial \varphi}{\partial x}(t, x), \varphi\right)=B_{1}(t, x, \varphi)+B^{1}\left(t, x, \frac{\partial \varphi}{\partial x}(t, x), \varphi\right)$.

Let us give the definition of viscosity solution which is introduced by Crandall and Lions [10] for the first order equation, then generalized to the second order by Gimbert and Lions [15].

Definition 6.1 Let $k=1, \ldots, \ell$, and $u^{(k)}$ be a continuous function.

(i) We say that $u^{(k)}$ is a viscosity supersolution (subsolution) of (6.1) if

$$
\begin{gathered}
\min \left\{r \varphi\left(t_{0}, x_{0}\right)-\frac{\partial \varphi}{\partial t}\left(t_{0}, x_{0}\right)-A\left(t_{0}, x_{0}, \frac{\partial \varphi}{\partial x}\left(t_{0}, x_{0}\right), \frac{\partial^{2} \varphi}{\partial x^{2}}\left(t_{0}, x_{0}\right)\right)-B\left(t_{0}, x_{0}, \frac{\partial \varphi}{\partial x}\left(t_{0}, x_{0}\right), \varphi\right) ;\right. \\
\left.\varphi\left(t_{0}, x_{0}\right)-\phi^{(k)}\left(t_{0}, x_{0}\right)\right\} \geq 0
\end{gathered}
$$

$(\leq 0)$ whenever $\varphi \in \mathcal{C}^{1,2}([0, T) \times \mathbb{R}) \cap \mathcal{C}_{2}([0, T] \times \mathbb{R})$ and $u^{(k)}-\varphi$ has a strict global minimum (maximum) at $\left(t_{0}, x_{0}\right) \in[0, T) \times \mathbb{R}$.

(ii) We say that $u^{(k)}$ is a viscosity solution of (6.1) if it is both super and subsolution of (6.1).

Theorem 6.1 For all $k=1, \ldots, \ell$, the value function $v^{(k)}$ is a viscosity solution of the $H J B V I(6.1)$ on $[0, T) \times \mathbb{R}$.

\section{Proof. Viscosity supersolution:}

Let $\varphi \in \mathcal{C}^{1,2}([0, T) \times \mathbb{R}) \cap \mathcal{C}_{2}([0, T] \times \mathbb{R})$ and $\left(t_{0}, x_{0}\right) \in[0, T) \times \mathbb{R}$ be a strict global minimum of $\varphi$ such that

$$
0=\left(v^{(k)}-\varphi\right)\left(t_{0}, x_{0}\right)=\min _{(t, x) \in[0, T) \times \mathbb{R}}\left(v^{(k)}-\varphi\right)(t, x) .
$$

Let $\eta>0$, we define the stopping time

$$
\theta:=\inf \left\{t>t_{0}:\left(t, X_{t}^{t_{0}, x_{0}}\right) \notin B_{\eta}\left(t_{0}, x_{0}\right)\right\} \wedge T
$$

where

$$
B_{\eta}\left(t_{0}, x_{0}\right):=\left\{(t, x) \in[0, T] \times \mathbb{R} \text { such that }\left|t-t_{0}\right|+\left|x-x_{0}\right| \leq \eta\right\} .
$$

Then from the Dynamic Programming Principle, it follows that

$$
\varphi\left(t_{0}, x_{0}\right)=v^{(k)}\left(t_{0}, x_{0}\right) \geq E^{Q}\left[e^{-r\left(\theta \wedge\left(t_{0}+h\right)\right)} \varphi\left(\theta \wedge\left(t_{0}+h\right), X_{\theta \wedge\left(t_{0}+h\right)}^{t_{0}, x_{0}}\right)\right] .
$$

By applying Itô's Lemma to $e^{-r s} \varphi\left(s, X_{s}^{t_{0}, x_{0}}\right)$ and using inequality (6.8) we obtain

$$
\begin{aligned}
0 & \geq E^{Q}\left[\frac { 1 } { h } \int _ { t _ { 0 } } ^ { \theta \wedge ( t _ { 0 } + h ) } e ^ { - r s } \left(-r \varphi\left(s, X_{s}^{t_{0}, x_{0}}\right)+\frac{\partial \varphi}{\partial s}\left(s, X_{s}^{t_{0}, x_{0}}\right)\right.\right. \\
& \left.\left.+A\left(s, X_{s}^{t_{0}, x_{0}}, \frac{\partial \varphi}{\partial x}\left(s, X_{s}^{t_{0}, x_{0}}\right), \frac{\partial^{2} \varphi}{\partial x^{2}}\left(s, X_{s}^{t_{0}, x_{0}}\right)\right)+B\left(s, X_{s}^{t_{0}, x_{0}}, \frac{\partial \varphi}{\partial x}\left(s, X_{s}^{t_{0}, x_{0}}\right), \varphi\right)\right) d s\right],
\end{aligned}
$$


where $A$ and $B$ are defined by (6.3) and (6.4) respectively. Sending $h$ to 0 , we deduce by the mean value theorem that

$$
\begin{aligned}
& \frac{1}{h} \int_{t_{0}}^{\theta \wedge\left(t_{0}+h\right)} e^{-r s}\left(\left(-r \varphi+\frac{\partial \varphi}{\partial s}\right)\left(s, X_{s}^{t_{0}, x_{0}}\right)+A\left(s, X_{s}^{t_{0}, x_{0}}, \frac{\partial \varphi}{\partial x}\left(s, X_{s}^{t_{0}, x_{0}}\right), \frac{\partial^{2} \varphi}{\partial x^{2}}\left(s, X_{s}^{t_{0}, x_{0}}\right)\right)\right. \\
& \left.\quad+B\left(s, X_{s}^{t_{0}, x_{0}}, \frac{\partial \varphi}{\partial x}\left(s, X_{s}^{t_{0}, x_{0}}\right), \varphi\right)\right) d s \longrightarrow \\
& e^{-r t_{0}}\left(\left(-r \varphi+\frac{\partial \varphi}{\partial s}\right)\left(t_{0}, x_{0}\right)+A\left(t_{0}, x_{0}, \frac{\partial \varphi}{\partial x}\left(t_{0}, x_{0}\right), \frac{\partial^{2} \varphi}{\partial x^{2}}\left(t_{0}, x_{0}\right)\right)+B\left(t_{0}, x_{0}, \frac{\partial \varphi}{\partial x}\left(t_{0}, x_{0}\right), \varphi\right)\right) .
\end{aligned}
$$

Then it follows from assumption (4.2) and the dominated convergence theorem that

$r \varphi\left(t_{0}, x_{0}\right)-\frac{\partial \varphi}{\partial s}\left(t_{0}, x_{0}\right)-A\left(t_{0}, x_{0}, \frac{\partial \varphi}{\partial x}\left(t_{0}, x_{0}\right), \frac{\partial^{2} \varphi}{\partial x^{2}}\left(t_{0}, x_{0}\right)\right)-B\left(t_{0}, x_{0}, \frac{\partial \varphi}{\partial x}\left(t_{0}, x_{0}\right), \varphi\right) \geq 0$

then $v^{(k)}$ is a viscosity supersolution of (6.1).

\section{Viscosity subsolution:}

Let $\varphi \in \mathcal{C}^{1,2}([0, T) \times \mathbb{R}) \cap \mathcal{C}_{2}([0, T] \times \mathbb{R})$, and $\left(t_{0}, x_{0}\right) \in[0, T) \times \mathbb{R}$ such that

$$
0=\left(v^{(k)}-\varphi\right)\left(t_{0}, x_{0}\right)>\left(v^{(k)}-\varphi\right)(t, x) \quad \text { for all }(t, x) \in[0, T) \times \mathbb{R} \backslash\left\{\left(t_{0}, x_{0}\right)\right\},
$$

then there exits $\eta>0$ such that

$$
\text { for all }(t, x) \notin B_{\eta}\left(t_{0}, x_{0}\right) \quad\left(v^{(k)}-\varphi\right)(t, x) \leq-\xi,
$$

where $B_{\eta}\left(t_{0}, x_{0}\right)$ is defined in $(6.7)$ and $\xi>0$.

In order to prove the required result, we assume to the contrary that there exists $\varepsilon>0$ such that for all $(t, x) \in B_{\eta}\left(t_{0}, x_{0}\right)$

$$
\min \left\{-\frac{\partial \varphi}{\partial t}(t, x)-\mathcal{L} \varphi(t, x) ; \varphi(t, x)-\phi^{(k)}(t, x)\right\} \geq \varepsilon,
$$

where $\mathcal{L} \varphi(t, x):=-r \varphi(t, x)+A\left(t, x, \frac{\partial \varphi}{\partial x}(t, x), \frac{\partial^{2} \varphi}{\partial x^{2}}(t, x)\right)+B\left(t, x, \frac{\partial \varphi}{\partial x}(t, x), \varphi\right)$.

Let us define the stopping times:

$$
\begin{aligned}
& \theta_{k}^{1}:=\inf \left\{t>t_{0}:\left(t, X_{t}^{t_{0}, x_{0}}\right) \notin B_{\eta}\left(t_{0}, x_{0}\right)\right\} \wedge T \\
& \theta_{k}^{2}:=\inf \left\{t>t_{0}: \phi^{(k)}\left(t, X_{t}^{t_{0}, x_{0}}\right)=v^{(k)}\left(t, X_{t}^{t_{0}, x_{0}}\right)\right\} \wedge T .
\end{aligned}
$$

On the set $\left\{\theta_{k}^{1} \leq \theta_{k}^{2}\right\}$ :

We have that $\left(\theta_{k}^{1}, X_{\theta_{k}^{1}}^{t_{0}, x_{0}}\right) \notin B_{\eta}\left(t_{0}, x_{0}\right)$, then by (6.10) we deduce that $v^{(k)}\left(\theta_{k}^{1}, X_{\theta_{k}^{1}}^{t_{0}, x_{0}}\right)+\xi \leq$ $\varphi\left(\theta_{k}^{1}, X_{\theta_{k}^{1}}^{t_{0}, x_{0}}\right)$. By applying Itô's Lemma to $e^{-r\left(s-t_{0}\right)} \varphi\left(s, X_{s}^{t_{0}, x_{0}}\right)$, we obtain

$$
\begin{aligned}
& e^{-r\left(\theta_{k}^{1}-t_{0}\right)}\left(v^{(k)}\left(\theta_{k}^{1}, X_{\theta_{k}^{1}}^{t_{0}, x_{0}}\right)+\xi\right)-v^{(k)}\left(t_{0}, x_{0}\right) \\
& \leq \int_{t_{0}}^{\theta_{k}^{1}} e^{-r\left(s-t_{0}\right)}\left(\frac{\partial \varphi}{\partial s}\left(s, X_{s}^{t_{0}, x_{0}}\right)+\mathcal{L} \varphi\left(s, X_{s}^{t_{0}, x_{0}}\right)\right) d s \\
& +\int_{t_{0}}^{\theta_{k}^{1}} e^{-r\left(s-t_{0}\right)} \sigma\left(s, X_{s}^{t_{0}, x_{0}}\right) \frac{\partial \varphi}{\partial x}\left(s, X_{s}^{t_{0}, x_{0}}\right) d W_{s} \\
& +\int_{t_{0}}^{\theta_{k}^{1}} e^{-r\left(s-t_{0}\right)} \int_{\mathbb{R} \backslash\{0\}}\left[\varphi\left(s, X_{s^{-}}^{t_{0}, x_{0}}+\gamma\left(s, X_{s^{-}}^{t_{0}, x_{0}}, z\right)\right)-\varphi\left(s, X_{s^{-}}^{t_{0}, x_{0}}\right)\right] \tilde{v}(d s, d z) .
\end{aligned}
$$


On the set $\left\{\theta_{k}^{1}>\theta_{k}^{2}\right\}$ :

We have that $\left(\theta_{k}^{2}, X_{\theta_{k}^{2}}^{t_{0}, x_{0}}\right) \in B_{\eta}\left(t_{0}, x_{0}\right)$, so by (6.11) and by applying Itô's Lemma to $e^{-r\left(s-t_{0}\right)} \varphi\left(s, X_{s}^{t_{0}, x_{0}}\right)$ we obtain

$$
\begin{aligned}
e^{-r\left(\theta_{k}^{2}-t_{0}\right)} & \left(\varepsilon+\phi^{(k)}\left(\theta_{k}^{2}, X_{\theta_{k}^{2}}^{t_{0}, x_{0}}\right)\right)-v^{(k)}\left(t_{0}, x_{0}\right) \\
\leq & \int_{t_{0}}^{\theta_{k}^{2}} e^{-r\left(s-t_{0}\right)}\left(\frac{\partial \varphi}{\partial s}\left(s, X_{s}^{t_{0}, x_{0}}\right)+\mathcal{L} \varphi\left(s, X_{s}^{t_{0}, x_{0}}\right)\right) d s \\
& +\int_{t_{0}}^{\theta_{k}^{2}} e^{-r\left(s-t_{0}\right)} \sigma\left(s, X_{s}^{t_{0}, x_{0}}\right) \frac{\partial \varphi}{\partial x}\left(s, X_{s}^{t_{0}, x_{0}}\right) d W_{s} \\
& +\int_{t_{0}}^{\theta_{k}^{2}} e^{-r\left(s-t_{0}\right)} \int_{\mathbb{R} \backslash\{0\}}\left[\varphi\left(s, X_{s^{-}}^{t_{0}, x_{0}}+\gamma\left(s, X_{s^{-}}^{t_{0}, x_{0}}, z\right)\right)-\varphi\left(s, X_{s^{-}}^{t_{0}, x_{0}}\right)\right] \tilde{v}(d s, d z) .
\end{aligned}
$$

Let us denote by $\theta_{k}:=\theta_{k}^{1} \wedge \theta_{k}^{2}$, then using inequalities (6.11), (6.12) and (6.13) we obtain

$$
\begin{aligned}
& \varepsilon E^{Q}\left[e^{-r\left(\theta_{k}^{2}-t_{0}\right)} \mathbf{1}_{\left\{\theta_{k}^{1}>\theta_{k}^{2}\right\}}\right]+E^{Q}\left[e^{-r\left(\theta_{k}^{1}-t_{0}\right)} v^{(k)}\left(\theta_{k}^{1}, X_{\theta_{k}^{1}}^{t_{0}, x_{0}}\right) \mathbf{1}_{\left\{\theta_{k}^{1} \leq \theta_{k}^{2}\right\}}\right] \\
& -v^{(k)}\left(t_{0}, x_{0}\right)+\xi E^{Q}\left[e^{-r\left(\theta_{k}^{1}-t_{0}\right)} \mathbf{1}_{\left\{\theta_{k}^{1} \leq \theta_{k}^{2}\right\}}\right]+E^{Q}\left[e^{-r\left(\theta_{k}^{2}-t_{0}\right)} \phi^{(k)}\left(\theta_{k}^{2}, X_{\theta_{k}^{2}}^{t_{0}, x_{0}}\right) \mathbf{1}_{\left\{\theta_{k}^{1}>\theta_{k}^{2}\right\}}\right] \\
& \leq-\varepsilon E^{Q}\left[\int_{t_{0}}^{\theta_{k}} e^{-r\left(s-t_{0}\right)} d s\right]<0 .
\end{aligned}
$$

Let us suppose that for all $\xi^{\prime}>0$, we have that

$$
H:=\xi E^{\mathcal{Q}}\left[e^{-r\left(\theta_{k}^{1}-t_{0}\right)} \mathbf{1}_{\left\{\theta_{k}^{1} \leq \theta_{k}^{2}\right\}}\right]+\varepsilon E^{\mathcal{Q}}\left[e^{-r\left(\theta_{k}^{2}-t_{0}\right)} \mathbf{1}_{\left\{\theta_{k}^{1}>\theta_{k}^{2}\right\}}\right] \leq \xi^{\prime},
$$

then

$$
0 \leq \xi E^{\mathcal{Q}}\left[e^{-r\left(\theta_{k}^{1}-t_{0}\right)} \mathbf{1}_{\left\{\theta_{k}^{1} \leq \theta_{k}^{2}\right\}}\right] \leq \xi^{\prime}
$$

By sending $\xi^{\prime}$ to 0 , we obtain that $\mathbf{1}_{\left\{\theta_{k}^{1} \leq \theta_{k}^{2}\right\}}=0$ a.s., then $\theta_{k}^{1}>\theta_{k}^{2}$ a.s. So we obtain that $H=\varepsilon E^{\mathcal{Q}}\left[e^{-r\left(\theta_{k}^{2}-t_{0}\right)}\right] \leq \xi^{\prime}$. By sending $\xi^{\prime}$ to 0 we obtain that $\varepsilon E^{\mathcal{Q}}\left[e^{-r\left(\theta_{k}^{2}-t_{0}\right)}\right] \leq 0$, which is in contradiction with the fact that $\varepsilon E^{\mathcal{Q}}\left[e^{-r\left(\theta_{k}^{2}-t_{0}\right)}\right]>0$. We conclude then that there exists $\xi^{\prime}>0$ such that $H \geq \xi^{\prime}$.

On the other hand, we have that $\left\{e^{-r t} v^{(k)}\left(t, X_{t}^{t_{0}, x_{0}}\right), t_{0} \leq t \leq T\right\}$ is a supermartingale, then by [16, Theorem D.9, p.355], the stopped supermartingale

$\left\{e^{-r\left(t \wedge \theta_{k}^{2}\right)} v^{(k)}\left(t \wedge \theta_{k}^{2}, X_{t \wedge \theta_{k}^{2}}^{t_{0}, x_{0}}\right), t_{0} \leq t \leq T\right\}$ is a martingale. From the growth condition on $v^{(k)}$, the martingale $\left\{e^{-r\left(t \wedge \theta_{k}^{2}\right)} v^{(k)}\left(t \wedge \theta_{k}^{2}, X_{t \wedge \theta_{k}^{2}}^{t_{0}, x_{0}}\right), t_{0} \leq t \leq T\right\}$ is bounded in $L^{1}(Q)$ and so uniformly integrable. By Doob's optional Stopping Theorem (see [24, Theorem 18, p.10]), we obtain that

$$
e^{-r t_{0}} v^{(k)}\left(t_{0}, x_{0}\right)=E^{Q}\left[e^{-r\left(t \wedge \theta_{k}\right)} v^{(k)}\left(t \wedge \theta_{k}, X_{t \wedge \theta_{k}}^{t_{0}, x_{0}}\right)\right] \quad \forall t \in \mathcal{S}_{t_{0}},
$$


and so

$$
\begin{aligned}
v^{(k)}\left(t_{0}, x_{0}\right) & =E^{Q}\left[e^{-r\left(\theta_{k}^{1}-t_{0}\right)} v^{(k)}\left(\theta_{k}^{1}, X_{\theta_{k}^{1}}^{t_{0}, x_{0}}\right) \mathbf{1}_{\left\{\theta_{k}^{1} \leq \theta_{k}^{2}\right\}}\right] \\
& +E^{Q}\left[e^{-r\left(\theta_{k}^{2}-t_{0}\right)} \phi^{(k)}\left(\theta_{k}^{2}, X_{\theta_{k}^{2}}^{t_{0}, x_{0}}\right) \mathbf{1}_{\left\{\theta_{k}^{1}>\theta_{k}^{2}\right\}}\right] .
\end{aligned}
$$

From inequality (6.14) and the fact that $H \geq \xi^{\prime}$, we deduce that $\xi^{\prime} \leq 0$, which contradicts the fact that $\xi^{\prime}>0$.

We conclude then that the value function $v^{(k)}$ is a viscosity subsolution of the equation $(6.1)$ on $[0, T) \times \mathbb{R}$.

The last theorem prove the uniqueness of viscosity solutions.

\section{Theorem 6.2 (Comparison Theorem)}

Assume that the assumptions (4.2), (4.4), (4.7) and the Lipschitz continuity of $\phi$ hold. Let $u^{(k)}$ (resp. $\left.v^{(k)}\right), k=1, \ldots, \ell$, be a viscosity subsolution (resp. supersolution) of (6.1). Assume also that $u^{(k)}$ and $v^{(k)}$ are Lipschitz, have a linear growth in $x$ and holder in $t$. If

$$
u^{(k)}(T, x) \leq v^{(k)}(T, x) \quad \forall x \in \mathbb{R},
$$

then

$$
u^{(k)}(t, x) \leq v^{(k)}(t, x) \quad \forall(t, x) \in[0, T] \times \mathbb{R}
$$

The proof is similar to the one given by Pham [23]. In our case we need to use an induction argument.

\section{Conclusion}

In this paper we generalized the results of Carmona and Touzi from continuous to right continuous and left continuous in expectation processes. In the second part we characterized the value function of the multiple stopping problem for a jump diffusion process as the unique viscosity solution of a sequence of HJBVI.

We can solve numerically the sequence of the HJBVI by using a discrete time scheme and the quantization method to compute the conditional expectations appearing in this scheme. The convergence of such scheme could be achieved by using viscosity solution arguments. This work is postponed in a further research.

\section{Appendix}

We recall the classical following theorem (see for example Karatzas Shreve (1998)). 
Theorem 8.1 (Essential supremum) Let $(\Omega, \mathcal{F}, \mathbb{P})$ be a probability space and let $\mathcal{X}$ be a non empty family of nonnegative random variables defined on $(\Omega, \mathcal{F}, \mathbb{P})$. Then there exists a random variable $X^{*}$ satisfying

1. for all $X \in \mathcal{X}, X \leq X^{*}$ a.s. ,

2. if $Y$ is a random variable satisfying $X \leq Y$ a.s. for all $X \in \mathcal{X}$, then $X^{*} \leq Y$ a.s..

This random variable, which is unique a.s., is called the essential supremum of $X$ and is denoted ess $\sup _{X \in \mathcal{X}} X$. Furthermore, if $\mathcal{X}$ is closed under pairwise maximization (that is: $X, Y \in \mathcal{X}$ implies $X \vee Y \in \mathcal{X})$, then there is a nondecreasing sequence $\left(Z_{n}\right)_{n \in \mathbb{N}}$ of random variable in $\mathcal{X}$ satisfying $X^{*}=\lim _{n \rightarrow \infty} Z_{n}$ almost surely.

Lemma 8.1 For all $0 \leq t<s \leq T$, there exists a constant $C>0$ such that

$$
E^{Q}\left[\left|X_{t+\delta}^{t, x}-X_{s+\delta}^{s, x}\right|\right] \leq C(1+|x|) \sqrt{s-t}
$$

Proof. Let $X^{1}$ and $X^{2}$ two processes such that

$$
\begin{aligned}
& \left\{\begin{array}{l}
X_{t}^{1}=x \\
d X_{u}^{1}=b\left(u, X_{u^{-}}^{1}\right) d u+\sigma\left(u, X_{u^{-}}^{1}\right) d W_{u}+\int_{\mathbb{R}} \gamma\left(u, X_{u^{-}}^{1}, z\right) \tilde{v}(d u, d z) \quad \forall u \in(t, t+\delta] \\
d X_{u}^{1}=0 \quad \forall u \in(t+\delta, s+\delta]
\end{array}\right. \\
& \left\{\begin{array}{l}
d X_{u}^{2}=0 \quad \forall u \in[t, s] \\
X_{s}^{2}=x \\
d X_{u}^{2}=b\left(u, X_{u^{-}}^{2}\right) d u+\sigma\left(u, X_{u^{-}}^{2}\right) d W_{u}+\int_{\mathbb{R}} \gamma\left(u, X_{u^{-}}^{2}, z\right) \tilde{v}(d u, d z) \quad \forall u \in(s, s+\delta]
\end{array}\right.
\end{aligned}
$$

We define $Y_{u}:=X_{u}^{1}-X_{u}^{2}$, then $Y_{t}=0$.

First case: $s<t+\delta$

$$
\begin{array}{cc}
d Y_{u}=b\left(u, X_{u^{-}}^{1}\right) d u & +\sigma\left(u, X_{u^{-}}^{1}\right) d W_{u}+\int_{\mathbb{R}} \gamma\left(u, X_{u^{-}}^{1}, z\right) \tilde{v}(d u, d z), \quad \forall u \in(t, s] \\
d Y_{u}=\left(b\left(u, X_{u^{-}}^{1}\right)-b\left(u, X_{u^{-}}^{2}\right)\right) d u+\left(\sigma\left(u, X_{u^{-}}^{1}\right)-\sigma\left(u, X_{u^{-}}^{2}\right)\right) d W_{u} & \\
+\int_{\mathbb{R}}\left(\gamma\left(u, X_{u^{-}}^{1}, z\right)-\gamma\left(u, X_{u^{-}}^{2}, z\right)\right) \tilde{v}(d u, d z), \quad \forall u \in(s, t+\delta] \\
d Y_{u}=-b\left(u, X_{u^{-}}^{2}\right) d u-\sigma\left(u, X_{u^{-}}^{2}\right) d W_{u}-\int_{\mathbb{R}} \gamma\left(u, X_{u^{-}}^{2}, z\right) \tilde{v}(d u, d z), \quad \forall u \in(t+\delta, s+\delta] .
\end{array}
$$


We obtain then in addition to the Lipschitz continuity and the linear growth condition in $x$ of $b, \sigma$ and $\gamma$ that

$$
\begin{aligned}
E^{Q}\left[\left|Y_{s+\delta}\right|^{2}\right] & \leq C E^{Q}\left[\int_{t}^{s}\left(\left|b\left(u, X_{u}^{1}\right)\right|^{2}+\left|\sigma\left(u, X_{u}^{1}\right)\right|^{2}+\int_{\mathbb{R}}\left|\gamma\left(u, X_{u}^{1}, z\right)\right|^{2} m(d z)\right) d u\right] \\
& +C E^{Q}\left[\int _ { s } ^ { t + \delta } \left(\left|b\left(u, X_{u}^{1}\right)-b\left(u, X_{u}^{2}\right)\right|^{2}+\left|\sigma\left(u, X_{u}^{1}\right)-\sigma\left(u, X_{u}^{2}\right)\right|^{2}\right.\right. \\
& \left.\left.+\int_{\mathbb{R}}\left|\gamma\left(u, X_{u}^{1}, z\right)-\gamma\left(u, X_{u}^{2}, z\right)\right|^{2} m(d z)\right) d u\right] \\
& +C E^{Q}\left[\int_{t+\delta}^{s+\delta}\left(\left|b\left(u, X_{u}^{2}\right)\right|^{2}+\left|\sigma\left(u, X_{u}^{2}\right)\right|^{2}+\int_{\mathbb{R}}\left|\gamma\left(u, X_{u}^{2}, z\right)\right|^{2} m(d z)\right) d u\right] .
\end{aligned}
$$

From Lemma 3.1 of Pham [23] and assumption (4.2), we deduce that

$$
E^{Q}\left[\left|Y_{s+\delta}\right|^{2}\right] \leq C\left((s-t)\left(1+|x|^{2}\right)+E^{Q}\left[\int_{t}^{s+\delta}\left|Y_{u}\right|^{2} d u\right]\right) .
$$

Second case: $t+\delta \leq s$

$$
\begin{array}{r}
d Y_{u}=b\left(u, X_{u^{-}}^{1}\right) d u+\sigma\left(u, X_{u^{-}}^{1}\right) d W_{u}+\int_{\mathbb{R}} \gamma\left(u, X_{u^{-}}^{1}, z\right) \tilde{v}(d u, d z), \quad \forall u \in(t, t+\delta] \\
d Y_{u}=0, \quad \forall u \in(t+\delta, s] \\
d Y_{u}=-b\left(u, X_{u^{-}}^{2}\right) d u-\sigma\left(u, X_{u^{-}}^{2}\right) d W_{u}-\int_{\mathbb{R}} \gamma\left(u, X_{u^{-}}^{2}, z\right) \tilde{v}(d u, d z), \quad \forall u \in(s, s+\delta] .
\end{array}
$$

We obtain then in addition to the linear growth conditions in $x$ of $b, \sigma$ and $\gamma$ that

$$
\begin{aligned}
E^{Q}\left[\left|Y_{s+\delta}\right|^{2}\right] & \leq C E^{Q}\left[\int_{t}^{t+\delta}\left(\left|b\left(u, X_{u}^{1}\right)\right|^{2}+\left|\sigma\left(u, X_{u}^{1}\right)\right|^{2}+\int_{\mathbb{R}}\left|\gamma\left(u, X_{u}^{1}, z\right)\right|^{2} m(d z)\right) d u\right] \\
& +C E^{Q}\left[\int _ { s } ^ { s + \delta } \left(\left|b\left(u, X_{u}^{1}\right)-b\left(u, X_{u}^{2}\right)\right|^{2}+\left|\sigma\left(u, X_{u}^{1}\right)-\sigma\left(u, X_{u}^{2}\right)\right|^{2} .\right.\right.
\end{aligned}
$$

From Lemma 3.1 of Pham [23] and assumption (4.2), we deduce that

$$
E^{Q}\left[\left|Y_{s+\delta}\right|^{2}\right] \leq C\left((s-t)\left(1+|x|^{2}\right)+E^{Q}\left[\int_{t}^{s+\delta}\left|Y_{u}\right|^{2} d u\right]\right) .
$$

We deduce then that in both cases we have that

$$
\leq C\left((s-t)\left(1+|x|^{2}\right)+E^{Q}\left[\int_{t}^{s+\delta}\left|Y_{u}\right|^{2} d u\right]\right) .
$$

Then by Fubini's theorem and by Gronwall's lemma we deduce that

$$
E^{Q}\left[\left|X_{s+\delta}^{s, x}-X_{t+\delta}^{t, x}\right|\right] \leq C(1+|x|) \sqrt{s-t} .
$$

We recall the theorem 2.18 of El Karoui [13]. 
Theorem 8.2 Let $X$ be a right continuous, nonnegative, bounded, adapted and LCE. Let $Y$ be the Snell envelope of $X$, i.e. the smallest right continuous supermartingale wich dominate $X$, it is defined by

$$
Y_{t}:=e s s \sup _{u \in \mathcal{S}_{t}} E\left[X_{u} \mid \mathcal{F}_{t}\right] \text { a.s. }
$$

The stopping time $D_{t}:=\inf \left\{u \geq t, X_{u}=Y_{u}\right.$ a.s. $\}$ is an optimal stopping time of (8.1), i.e.

$$
Y_{t}:=e s s \sup _{u \in \mathcal{S}_{t}} E\left[X_{u} \mid \mathcal{F}_{t}\right]=E\left[X_{D_{t}} \mid \mathcal{F}_{t}\right] \text { a.s.. }
$$

To prove this Theorem we need to the following Theorem and Proposition.

Theorem 8.3 Let $\left(X_{t}\right)_{t}$ be a nonnegative right continuous supermartingale. If $\sigma, \tau$ are stopping times with $\sigma \leq \tau$, we have $E\left[X_{\tau} \mid \mathcal{F}_{\sigma}\right] \leq X_{\sigma}$ a.s.

Proposition 8.1 For all $\lambda \in(0,1)$, let $A^{\lambda}$ be the set $A_{t}^{\lambda}:=\left\{(\omega, s) ; s \geq t, X_{s}(\omega) \geq\right.$ $\left.\lambda Y_{s}(\omega)\right\}$, and $D_{t}^{\lambda}$ the first time of $A^{\lambda}$ greater than $t$, i.e. $D_{t}^{\lambda}:=\inf \left\{s \geq t ; X_{s} \geq \lambda Y_{s}\right\}$. Then,

$$
\forall t \in \mathcal{S}, \quad Y_{t}=E\left[Y_{D_{t}^{\lambda}} \mid \mathcal{F}_{t}\right] \text { a.s. }
$$

Proof. Since $X$ is a supermartingale and since $D_{t}^{\lambda} \geq t$ a.s. then we have

$$
E\left[Y_{D_{t}^{\lambda}} \mid \mathcal{F}_{t}\right] \leq Y_{t} \text { a.s. }
$$

It remains to show the reverse inequality. This will be done in two steps. For ease of notation, we denote by $U_{t}^{\lambda}:=E\left[Y_{D_{t}^{\lambda}} \mid \mathcal{F}_{t}\right]$.

Step 1: Let us prove that $\left(U_{\tau}^{\lambda}, \tau \in \mathcal{S}\right)$ is a supermartingale. Fix $\tau, \tau^{\prime} \in \mathcal{S}$ such that $\tau^{\prime} \geq \tau$ a.s., we have that $E\left[U_{\tau^{\prime}}^{\lambda} \mid \mathcal{F}_{\tau}\right]=E\left[E\left[Y_{D_{\tau^{\prime}}^{\lambda}} \mid \mathcal{F}_{D_{\tau}^{\lambda}}\right] \mid \mathcal{F}_{\tau}\right]=E\left[Y_{D_{\tau^{\prime}}^{\lambda}} \mid \mathcal{F}_{\tau}\right]$ a.s. From the right continuity of $X$ and $Y$ we have that $A_{t}^{\lambda}$ is nonincreasing then $D_{\tau^{\prime}}^{\lambda} \geq D_{\tau}^{\lambda}$ a.s. Since $\left(Y_{t}\right)$ is a supermartingale then $E\left[Y_{D_{\tau^{\prime}}^{\lambda}} \mathcal{F}_{D_{\tau}^{\lambda}}\right] \leq Y_{D_{\tau}^{\lambda}}$ a.s. Consequently, $E\left[U_{\tau^{\prime}}^{\lambda} \mid \mathcal{F}_{\tau}\right] \leq E\left[Y_{D_{\tau}^{\lambda}} \mid \mathcal{F}_{\tau}\right]=U_{\tau}^{\lambda}$ a.s; which ends the proof of step 1 .

Step 2: Let us show that for each $\tau \in \mathcal{S}$ and each $\lambda \in(0,1)$,

$$
\lambda Y_{\tau}+(1-\lambda) U_{\tau}^{\lambda} \geq X_{\tau} \text { a.s. }
$$

Fix $\tau \in \mathcal{S}$ and $\lambda \in(0,1)$. We have that on $B:=\left\{X_{\tau} \geq \lambda Y_{\tau}\right\}, D_{\tau}^{\lambda}=\tau$ a.s., then $U_{\tau}^{\lambda}:=E\left[Y_{D_{\tau}^{\lambda}} \mid \mathcal{F}_{\tau}\right]=E\left[Y_{\tau} \mid \mathcal{F}_{\tau}\right]=Y_{\tau}$ a.s. on $B$. This implies the inequality

$$
\lambda Y_{\tau}+(1-\lambda) U_{\tau}^{\lambda}=Y_{\tau} \geq X_{\tau} \text { a.s. on } B \text {. }
$$


Furthermore, since $B^{c}:=\left\{X_{\tau}<\lambda Y_{\tau}\right\}$ and since $U_{\tau}^{\lambda}$ is nonnegative, then

$$
\lambda Y_{\tau}+(1-\lambda) U_{\tau}^{\lambda} \geq \lambda Y_{\tau} \geq X_{\tau} \text { a.s. on } B^{c} .
$$

The proof of step 2 is complete.

By step 1 and the supermartingale property of $Y$ we obtain that $\left\{\lambda Y_{\tau}+(1-\lambda) U_{\tau}^{\lambda}, \tau \in \mathcal{S}\right\}$ is a supermartingale. By step 2, it dominates $\left\{X_{\tau}, \tau \in \mathcal{S}\right\}$. Consequently,

by the characterization of $Y$ as the smallest supermartingale which dominates $X$, we have

$$
\lambda Y_{\tau}+(1-\lambda) U_{\tau}^{\lambda} \geq Y_{\tau} \text { a.s. }
$$

Hence, $U_{\tau}^{\lambda} \geq Y_{\tau}$ a.s. (note that the strict inequality $\lambda<1$ is necessary here). We conclude that for each $\tau \in \mathcal{S}, E\left[Y_{D_{\tau}^{\lambda}} \mid \mathcal{F}_{\tau}\right]:=U_{\tau}^{\lambda}=Y_{\tau}$ a.s.

Let us now prove Theorem 8.2.

Proof. Let $\left(\lambda_{n}\right)$ be a nondecreasing sequence of reals valued in $(0,1)$, such that $\lim _{n \rightarrow \infty} \lambda_{n}=1$. It is clear that the sets $A_{t}^{\lambda_{n}}$ are nonincreasing and $D_{t}^{\lambda_{n}}$ are nondecreasing. Since the map $\lambda \mapsto D_{t}^{\lambda}$ is nondecreasing on $(0,1)$, the random variable $\bar{D}_{t}$ defined by $\bar{D}_{t}:=\lim _{n \rightarrow \infty} D_{t}^{\lambda_{n}}$ is a stopping time. Let us prove that it is an optimal stopping time of (8.1), i.e. $Y_{t}:=\operatorname{ess} \sup _{u \in \mathcal{S}_{t}} E\left[X_{u} \mid \mathcal{F}_{t}\right]=E\left[X_{\bar{D}_{t}} \mid \mathcal{F}_{t}\right]$ a.s.

Since $X$ and $Y$ are right continuous then $D_{t}^{\lambda_{n}} \in A_{t}^{\lambda_{n}}$, i.e. $X_{D_{t}^{\lambda_{n}}} \geq \lambda_{n} Y_{D_{t}^{\lambda_{n}}}$. Hence using Proposition 8.1, we obtain $Y_{t}=E\left[Y_{D_{t}^{\lambda_{n}}} \mid \mathcal{F}_{t}\right] \leq \frac{1}{\lambda_{n}} E\left[X_{D_{t}^{\lambda_{n}}} \mid \mathcal{F}_{t}\right]$ a.s. and so $E\left[Y_{t}\right] \leq$ $\frac{1}{\lambda_{n}} E\left[X_{D_{t}^{\lambda_{n}}}\right]$. Passing to the limit as $n$ tends to $\infty$ and using the fact that $X$ is LCE we get $E\left[Y_{t}\right] \leq E\left[X_{\bar{D}_{t}}\right]$ and hence $E\left[Y_{t}\right]=E\left[X_{\bar{D}_{t}}\right]=E\left[E\left[X_{\bar{D}_{t}} \mid \mathcal{F}_{t}\right]\right]$. Since $Y_{t} \geq E\left[X_{\bar{D}_{t}} \mid \mathcal{F}_{t}\right]$ a.s., the last equality gives $Y_{t}=E\left[X_{\bar{D}_{t}} \mid \mathcal{F}_{t}\right]$ a.s. which prove the optimality of $\bar{D}_{t}$. It is clear that $\bar{D}_{t} \leq D_{t}$, since for all $n$ we have $\left\{u \geq t ; X_{u}=Y_{u}\right\} \subset A_{t}^{\lambda_{n}}$ then $D_{t}^{\lambda_{n}} \leq D_{t}$ and hence $\bar{D}_{t} \leq D_{t}$. Let us prove that $\bar{D}_{t} \geq D_{t}$. Since $\bar{D}_{t}$ is an optimal stopping time, then $E\left[X_{\bar{D}_{t}}\right]=E\left[Y_{t}\right]$. On the other hand, using $Y \geq X$ and applying Theorem 8.3 to the supermartingale $Y$ we obtain that $E\left[X_{\bar{D}_{t}}\right] \leq E\left[Y_{\bar{D}_{t}}\right] \leq E\left[Y_{t}\right]$. Therefore, $E\left[Y_{\bar{D}_{t}}\right]=E\left[X_{\bar{D}_{t}}\right]$. Since $Y_{\bar{D}_{t}} \geq X_{\bar{D}_{t}}$ then $Y_{\bar{D}_{t}}=X_{\bar{D}_{t}}$ a.s., so $\bar{D}_{t} \geq D_{t}$. We conclude that $D_{t}:=\inf \left\{u \geq t, X_{u}=Y_{u}\right\}$ is an optimal stopping time of (8.1).

Proposition 8.2 Let $\tau^{*}$ be an optimal stopping time of (8.1), then the stopped process $Y^{\tau^{*}}$ defined by $Y_{s}^{\tau^{*}}=Y_{\tau^{*} \wedge s}, t \leq s \leq T$ is a $\mathbb{P}-(\mathcal{F})_{t}$ martingale.

Proof. We have that $\tau^{*}$ is an optimal stopping time of (8.1) then $Y_{t}=E\left[X_{\tau^{*}} \mid \mathcal{F}_{t}\right]$ a.s. and hence $E\left[Y_{t}\right]=E\left[X_{\tau^{*}}\right]$. On the other hand, using $Y \geq X$ and the supermartingale property of $Y$, we have $E\left[X_{\tau^{*}}\right] \leq E\left[Y_{\tau^{*}}\right] \leq E\left[Y_{t}\right]$. Therefore,

$$
E\left[Y_{\tau^{*}}\right]=E\left[Y_{t}\right]
$$

From the supermartingale property of $Y$ we have that for all $s \in[t, T], E\left[Y_{\tau^{*}}\right] \leq E\left[Y_{\tau^{*} \wedge s}\right]$ and $E\left[Y_{t}\right] \geq E\left[Y_{\tau^{*} \wedge s}\right]$. Using equality (8.7) we obtain that $E\left[Y_{t}\right]=E\left[Y_{\tau^{*} \wedge s}\right]$ for all 
$s \in[t, T]$. So we have that for all $t \leq u \leq u^{\prime} \leq T, E\left[Y_{\tau^{*} \wedge u}\right]=E\left[Y_{\tau^{*} \wedge u^{\prime}}\right]$. From the supermartingale property of $Y$, we have that $E\left[Y_{\tau^{*} \wedge u^{\prime}} \mid \mathcal{F}_{u}\right] \leq Y_{\tau^{*} \wedge u}$ a.s., this inequality together with the last equality is equivalent to $Y_{\tau^{*} \wedge u}=E\left[Y_{\tau^{*} \wedge u^{\prime}} \mid \mathcal{F}_{u}\right]$ a.s. We obtain then the desired result.

\section{References}

[1] C. Bender (2011a). Primal and Dual Pricing of Multiple Exercise Options in Continuous Time. SIAM J. Finan. Math., 2, 562-586.

[2] C. Bender (2011b). Dual Pricing of Multi-Exercise Options under Volume Constraints. Finance Stoch, 15(1), 1-26.

[3] C. Bender, N. Dokuchaev (2014). A First Order BSPDE for Swing Option Pricing: Classical Solutions. (Preprint)

[4] C. Bender and J. Schoenmakers (2006). An Iterative Method for Multiple Stopping: Convergence and Stability. Adv. Appl. Probab., 38(3), 729-749.

[5] C. Bender, J. Schoenmakers, J. Zhang (2014). Dual Representations for General Multiple Stopping Problems. Math. Finance, early view.

[6] A. Bouzgenda Zeghal and M. Mnif (2006). Optimal Multiple stopping and valuation of Swing Options in Lévy Models. Int. J. Theor. Appl. Finance, 9(8):1267-1297.

[7] R. Carmona and S. Dayanik (2008). Optimal multiple stopping of linear diffusions. Mathematics of Operations Research,. (33):446460.

[8] R. Carmona and N. Touzi (2008). Optimal Multiple Stopping and valuation of Swing Options. Mathematical Finance, 18(2), 239268.

[9] M.G. Crandall and H. Ishii (1990). The maximum principle for semicontinuous functions, Diff. Int. Eqn., 3, 1001-1014.

[10] M.G. Crandall and P.L. Lions (1983). Viscosity solutions of Hamilton-Jacobi equations, Trans. A.M.S., 277, 1-42.

[11] M. Dahlgren and R. Korn. (2005). The swing option on the stock market,International Journal of Theoretical and Applied Finance, 8123139.

[12] C. Dellacherie and P. A. Meyer (1980). Probabilités et potentiel. Chapitres V à VIII : théorie des martingales. Hermann.

[13] N. El Karoui (1981). Les aspects probabilistes du controle stochastique, Lect. Notes in Math 876, 73-238. Springer verlag, New York N.Y. 
[14] N. El Karoui,J.-P. Lepeltier and A. Millet (1992). A probabilistic approach to the reduite in optimal stopping, prbability and mathematical statistics. vol. 13, Fbsc. 1, 97-121.

[15] F. Gimbert and P.L. Lions (1984). Existence and regularity results for solutions of second order integrodifferential operators, Ricerche Mat. XXXIII(2), 315-358.

[16] I. Karatzas and S.E. Shreve (1998). Methods of mathematical finance, Springer.

[17] M. Kobylanski, M.C. Quenez and E. Rouy-Mironescu (2009). Optimal multiple stopping time problem, to appear in Annals of Applied Probability.

[18] P.L Lions (1983). Optimal control of diffusion processes and Hamilton-JacobiBellman equations. Part 1: The dynamic programming principle and applications, and Part 2: viscosity solutions and uniqueness, Comm. P.D.E, 8, 1101-1174 and 1229-1276.

[19] M.A. Maingueneau (1978). Temps d'arrêt optimaux et theorie générale. Séminaire de probabilités (Strasbourg), tome 12, p.457-467.

[20] B. Øksendal and A.Sulem (2007). Applied Stochastic Control of Jump Diffusions. Springer.

[21] G. Peskir and A. Shiryaev, Optimal Stopping and Free-Boundary Problems (2006). Lectures in Mathematics ETH Zurich.

[22] H.Pham (1994). Optimal stopping and American option in jump diffusion model. Applied Mathematics and Optimization, vol 35, 145-164.

[23] H. Pham (1998). Optimal Stopping of Controlled Jump Diffusion Processes: A viscosity Solution Approach. Journal of Mathematical Systems, Estimation, and Control. vol 8, No. 1,pp. 1-2\%.

[24] P.E. Protter (2004). Stochastic integration and differential equations. Springerverlag Berlin Heidelberg.

[25] L.C.G. Rogers and D. Williams (2000). Diffusions, Markov Processes and Martingales. Volume 1 - Foundations. Cambridge University Press, second edition.

[26] A. Sayah (1991). Equations d'Hamilton-Jacobi du premier ordre avec terme intégrodifferentiels: Parties I et II, Comm. P.D.E., 10, 1057-1093.

[27] H.M. Soner (1986). Optimal control with state space constraint II, SIAM J.Control Optim., 24, 110-1122. 